Victor Lopes da Silva

\title{
Um formalismo de operadores de projeção para resolver o Hamiltoniano de Anderson
}

Dissertação apresentada como requisito parcial para obtenção do grau de Mestre pelo Programa de Pós-graduação em Física do Departamento de Física da PUC-Rio

Orientador : Prof. Enrique Victoriano Anda Co-Orientador: Prof. Ignacio Hamad 
Victor Lopes da Silva

\section{Um formalismo de operadores de projeção para resolver o Hamiltoniano de Anderson}

Dissertação apresentada como requisito parcial para obtenção do grau de Mestre pelo Programa de Pós-graduação em Física do Departamento de Física do Centro Técnico Científico da PUCRio. Aprovada pela comissão examinadora abaixo assinada.

Prof. Enrique Victoriano Anda

Orientador

Departamento de Física - PUC-Rio

Prof. Ignacio Hamad

Co-Orientador

Consejo Nacional de Investigaciones Científicas y Técnicas -

CONICET, Argentina

Prof. Edson Vernek

Universidade Federal de Uberlândia — UFU

Prof. Sergio Saul Makler

Universidade Federal de Juiz de Fora - UFJF

Prof. José Eugenio Leal

Coordenador Setorial do Centro Técnico Científico - PUC-Rio

Rio de Janeiro, 17 de Setembro de 2013 
Todos os direitos reservados. Proibida a reprodução total ou parcial do trabalho sem autorização da universidade, do autor e do orientador.

\section{Victor Lopes da Silva}

Graduou-se em Física na Universidade Federal do Rio de Janeiro - UFRJ.

Ficha Catalográfica

Lopes, Victor

Um formalismo de operadores de projeção para resolver o Hamiltoniano de Anderson / Victor Lopes da Silva; orientador: Enrique Victoriano Anda; co-orientador: Ignacio Hamad. Rio de Janeiro : PUC-Rio, Departamento de Física, 2013.

v., 59 f: il. ; $29,7 \mathrm{~cm}$

1. Dissertação (Mestrado em Física) - Pontifícia Universidade Católica do Rio de Janeiro, Departamento de Física.

Inclui referências bibliográficas.

1. Física - Tese. Hamiltoniano de Anderson; Efeito Kondo; Formalismo de Operadores de Projeção; Elétrons Fortemente Correlacionados I. Anda, Enrique. II. Hamad, Ignacio. III. Pontifícia Universidade Católica do Rio de Janeiro. Departamento de Física. IV. Título. 
A minha família. 


\section{Agradecimentos}

Primeiramente agradeço aos meus pais, José e Luciene, por terem se superado para que eu sempre pudesse ter a melhor educação dentro do possível. Ao meu irmão Felipe por ter sempre ter sido compreenssível e ao Popó por me acompanhar na minha jornada.

Aos professores do IF-UFRJ Felipe Pinheiro e Raimundo dos Santos, que apesar de não participarem deste trabalho, contribuíram para a minha formação como futuro pesquisador nos meus primeiros passos.

Ao meu grande amigo desde a graduação Amintor Dusko, que nas horas mais sombrias sempre contei com seu apoio e que, apesar dos caminhos diferentes que nossas vidas tomaram, os laços de amizade não foram rompidos.

A todo o Departamento de Física da PUC-Rio por ter me recebido de braços abertos, em especial aos professores Marco Cremona, Marcelo da Costa, Welles Morgado e Hiroshi Nunokawa por terem me ajudado nesta difícil transição.

Aos funcionários da PUC, em especial a Giza, sempre profissionais e solícitos.

Aos meus colegas de sala pelo excelente convívio e ambiente agradável.

A PUC-Rio e a CAPES pelo apoio financeiro.

Ao Ignácio Hamad sempre muito atencioso e com grandes sugestões ao trabalho.

Ao colaborador Pablo Roura Blas por suas valorosas dicas sobre programação e cálculo numérico.

Por fim, mas não menos importante, ao meu orientador Enrique Victoriano Anda e o grande responsável por este trabalho, por ter aceito me orientar e que a cada reunião sempre possuía algo a me ensinar. 


\section{Resumo}

Lopes, Victor; Anda, Enrique (orientador); Hamad, Ignacio. Um formalismo de operadores de projeção para resolver o Hamiltoniano de Anderson. Rio de Janeiro, 2013. 59p. Dissertação de Mestrado - Departamento de Física, Pontifícia Universidade Católica do Rio de Janeiro.

Nesta dissertação propomos um formalismo de operadores de projeção para obter a energia do estado fundamental do Hamiltoniano da Impureza de Anderson com repulsão Coulombiana $U$ infinita. Este formalismo consiste em projetar o espaço de Hilbert em um subespaço de uma única função correspondente ao estado fundamental do mar de Fermi, onde uma versão renormalizada do Hamiltoniano opera. A energia do estado fundamental pode ser obtida através de um processo autoconsistente. Conhecendo a energia é possível calcular as propriedades fundamentais do sistema como a magnetização em função do campo magnético externo, a susceptibilidade magnética, a dependência da ocupação eletrônica como função da energia local da impureza e a temperatura Kondo, a qual caracteriza o comportamento universal do problema Kondo.

\section{Palavras-chave}

Hamiltoniano de Anderson; Efeito Kondo; Formalismo de Operadores de Projeção; Elétrons Fortemente Correlacionados. 


\section{Abstract}

Lopes, Victor; Anda, Enrique (advisor); Hamad, Ignacio. A Projector Operator Formalism to solve the Anderson Hamiltonian. Rio de Janeiro, 2013. 59p. MSc. Dissertation Departamento de Física, Pontifícia Universidade Católica do Rio de Janeiro.

In this dissertation we propose a projector operator formalism to obtain the ground state energy of the Impurity Anderson Hamiltonian with infinite Coulomb repulsion $U$. This formalism consists in projecting the Hilbert space into a sub-space of one function corresponding to the ground state of the free Fermi sea where a renormalized version of the Hamiltonian operates. The ground state energy can be obtained through a self-consistent process. From the knowledge of the energy, it is possible to calculate the fundamental properties of the system as it is the magnetization as a function of an external magnetic field, the magnetic susceptibility, the dependence of the electronic occupation as a function of the local energy of the impurity and the Kondo temperature, which characterizes the universal behavior of a Kondo problem.

\section{Keywords}

Anderson Hamiltonian; Kondo Effect; Projector Operator Formalism; Strongly Correlated Electrons. 


\section{Sumário}

1 Formulação do Problema $\quad 12$

$\begin{array}{lll}1.1 & \text { Introdução } & 12\end{array}$

$\begin{array}{lll}1.2 & \text { Problema Kondo } & 14\end{array}$

$\begin{array}{lll}1.3 & \text { Pontos Quânticos } & 15\end{array}$

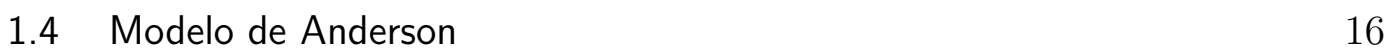

2 Determinação da energia do Hamiltoniano de Anderson utilizando um formalismo de operadores de projeção $\quad 20$

2.1 Introdução 20

2.2 Decompondo o espaço de Hilbert 21

2.3 Construção dos subespaços 23

2.4 Cálculo da correção da energia 27

2.5 Passagem ao contínuo 29

2.6 Banda plana e o comportamento da derivada 31

2.7 Função de Green de uma cadeia linear semi-infinita 33

2.8 Termos diagonais 34

2.9 Termos não diagonais 39

2.10 Resumo do capítulo 42

3 Solução Numérica $\quad 44$

$\begin{array}{lll}3.1 & \text { Algoritmo } & 44\end{array}$

3.2 Resultados 45

4 Conclusões e Perspectivas $\quad 56$

$\begin{array}{ll}\text { Referências Bibliográficas } & 58\end{array}$ 


\section{Lista de figuras}

Figura 1.1 Resistividade residual do Na devido a defeitos na rede e a interação elétron-fônon, como função da temperatura, (1).

Figura 1.2 Resistência elétrica para uma liga de cobre e ferro em diferentes concentrações. As barras indicam a localização do mínimo na resistência, (3).

Figura 1.3 Imagem feita com escaneamento de microscopia eletrônica, (6).

Figura 1.4 Condutância diferencial.

Figura 2.1 Decomposição do espaço de Hilbert em dois subespaços, $|1\rangle$ e $|2\rangle$.

Figura 2.2 Escolha do subespaço $|1\rangle$ como sendo o do mar de Fermi cheio e a impureza e a banda de condução vazias.

Figura 2.3 Construção do subespaço $|2\rangle$, representando esquematicamente três famílias de estados.

Figura 2.4 Cadeia linear semi-infinita.

Figura 2.5 Analogia dos subespaços $|1\rangle$ e $|2\rangle$ com a cadeia linear semiinfinita.

Figura 2.6 Rede de Bethe.

Figura 2.7 Termos não diagonais do subespaço $|2\rangle$. Os estados destacados são renormalizados devido à contribuição dos termos diagonais.

Figura 3.1 A linha contínua apresenta a parte real da função $\sum_{\sigma} f_{1 \sigma}(\xi)$ e a linha tracejada a $f(\xi)=\xi$. A intersecção entre as mesmas fornece a correção da energia $\Delta E$. Como buscamos a energia do estado fundamental, a intersecção que fornece a correção da energia é aquela de menor valor. $\mathrm{O}$ valor de $\eta$ utilizado é de $\eta=10^{-7}$.

Figura 3.2 Ampliação do gráfico da figura 3.1 na região de corte entre as funções parte real $\sum_{\sigma} f_{1 \sigma}(\xi)$ e $f(\xi)=\xi$.

Figura 3.3 A linha contínua apresenta parte real da função $f_{2 \sigma}(\xi)$ e a linha tracejada a função $f(\xi)=\xi-\epsilon_{0}$.

Figura 3.4 Gráfico que apresenta a intersecção da parte real $\sum_{\sigma} f_{1 \sigma}(\xi)$ com $f(\xi)=\xi$ e a intersecção da parte real $f_{2 \sigma}(\xi)$ e $f(\xi)=\xi-\epsilon_{0}$. A intersecção ocorre no mesmo ponto, $\xi=\Delta E$, correspondente a correção da energia.

Figura 3.5 Termo não diagonal.

Figura 3.6 Correção da energia como função da energia da impureza.

Figura 3.7 Número de ocupação eletrônica na impureza como função da energia da impureza.

Figura 3.8 Cálculo da correção da energia para diferentes valores do campo magnético externo e o ajuste polinomial. 
Figura 3.9 Magnetização da impureza como função do campo magnético externo. Obtida com derivada numérica para diferentes valores da energia da impureza.

Figura 3.10 Magnetização da impureza como função do campo magnético externo, na região em que a magnetização possuiu um comportamento linear, para os mesmos parâmetros da figura $3.9 \operatorname{com} \epsilon_{0}=-3$.

Figura 3.11 Evolução do logarítimo da razão da temperatura Kondo dividida pela semi-largura da banda, com a razão da energia da impureza dividida pela função de hibridização. 
"Protect me from knowing what I don't need to know. Protect me from even knowing that there are things to know that I don't know. Protect me from knowing that I decided not to know about the things that I decided not to know about. Amen."

Douglas Adams, Mostly Harmless. 


\section{Formulação do Problema}

\section{1}

\section{Introdução}

Em materiais não magnéticos condutores, a resistividade decresce monotonicamente com o decrécimo da temperatura $T$, pois as fontes do espalhamento dos elétrons de condução originam termos que são independentes da temperatura, a chamada resistividade residual em metais (regra de Mathiessen), figura 1.1. Numa visão heurística, isto pode ser entendido da seguinte forma: ao diminuírmos a temperatura, as vibrações da rede cristalina diminuem e, por conseguinte, a resistividade atinge um valor residual proveniente das imperfeições ou impurezas da rede, fontes de espalhamento dos elétrons.

Em contrapartida, em materiais com impurezas magnéticas os momentos magnéticos localizados no meio acoplam-se com os elétrons de condução e atuam como centros espalhadores em que o spin do elétron espalhado é trocado juntamente com o spin do momento local. Portanto, nestes materiais temos duas formas de espalhamento que competem, a interação elétron-fônon e o acoplamento magnético dos elétrons de condução com os momentos magnéticos do meio. A temperaturas altas, a principal contribuição para a resistividade é o espalhamento devido à interação elétron-fônon. Já a baixas temperaturas a principal contribuição para resistividade elétrica é a interação magnética entre o spin local e os spins dos elétrons da banda de condução do metal, (1) e (2).

Em ligas magnéticas diluídas, sabe-se desde 1930 que em vez da resistividade cair monotonicamente com a temperatura, a resistividade apresenta um mínimo a temperaturas da ordem de $10 \mathrm{~K}$ que depende fracamente da concentração de impurezas magnéticas, conforme a figura 1.2.

Tipicamente, impurezas magnéticas diluídas numa matriz metálica 
Um formalismo de operadores de projeção para resolver o Hamiltoniano de Ani

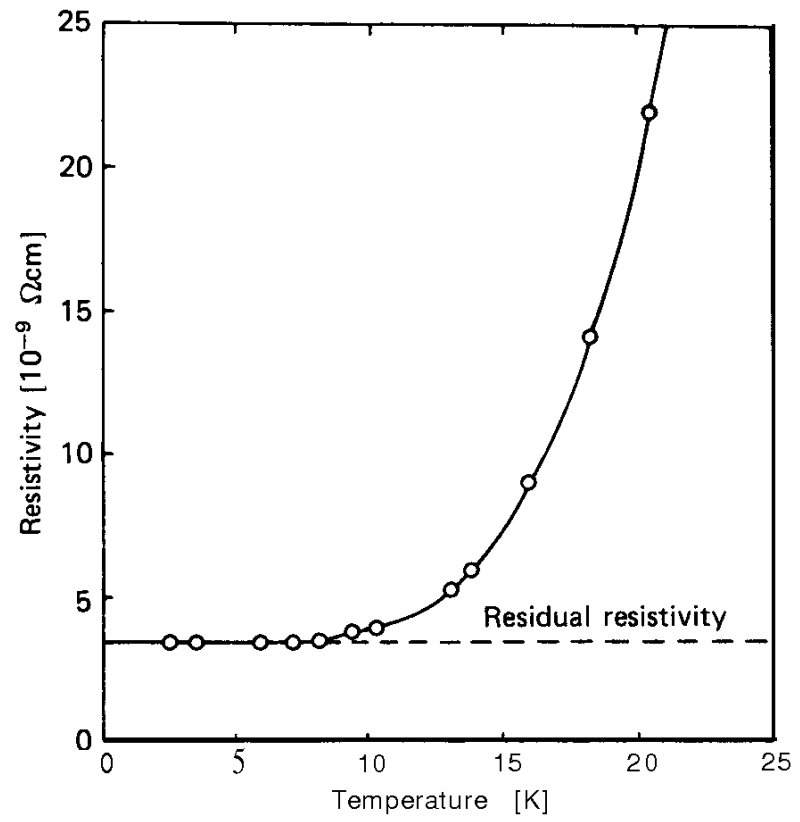

Figura 1.1: Resistividade residual do Na devido a defeitos na rede e a interação elétron-fônon, como função da temperatura, (1).

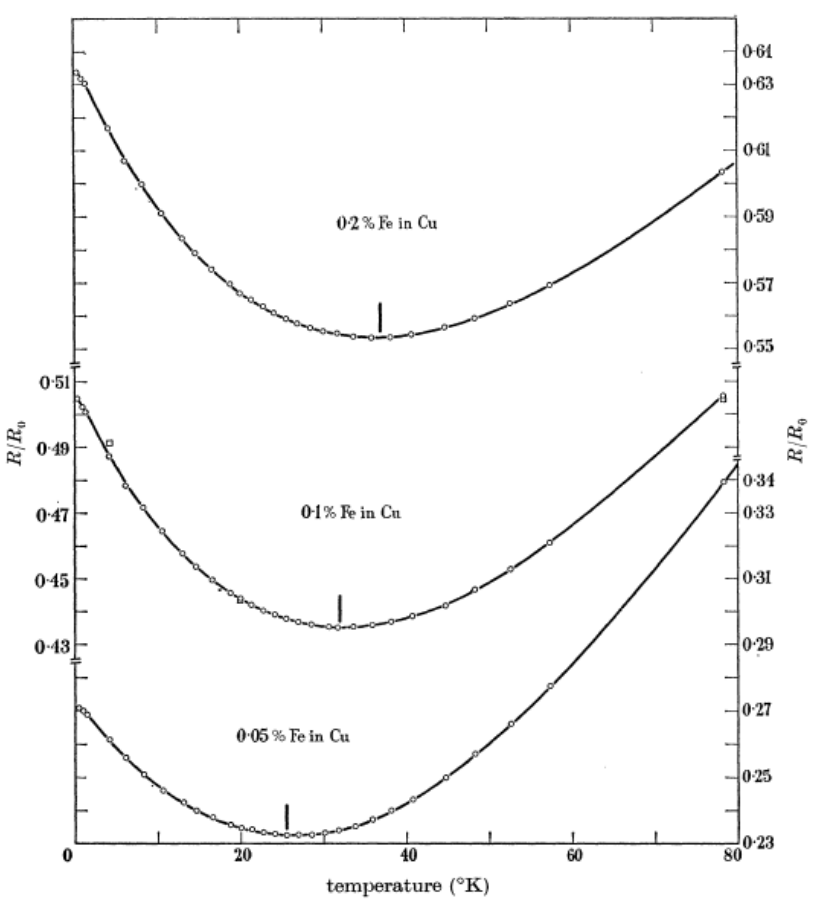

Figura 1.2: Resistência elétrica para uma liga de cobre e ferro em diferentes concentrações. As barras indicam a localização do mínimo na resistência, (3).

apresentam esse comportamento. São metais de transição 3d, bem como terras 
raras $4 \mathrm{f}$ e tem como exemplos bem conhecidos impurezas de $\mathrm{Fe}$ em um meio de $C u$, figura 1.2, e $C e$ em $L a A l_{2}$ e em $L a B_{6}$. Experimentalmente, é observado que estas impurezas dão contribuições anômalas às propriedades metálicas, especialmente às propriedades de transporte, como resistividade e o coeficiente Seebeck, bem como ao comportamento termodinâmico, (4).

\section{2}

\section{Problema Kondo}

Em 1964 J. Kondo elaborou uma explicação para a presença do mínimo na resistividade que resulta do espalhamento dos elétrons de condução próximos ao nível de Fermi pelos momentos magnéticos presentes no meio (5). Antes da explicação proposta por Kondo, o espalhamento era tratado principalmente elevando-se a ordem das teorias de pertubação, mas sem incluir novas interações. Kondo mostrou, usando teoria de perturbação de terceira ordem, que o acoplamento magnético leva a uma singularidade na secção de choque dos elétrons de condução próximos ao nível de Fermi e a uma dependência logarítimica com a temperatura na resistividade. Tal termo, juntamente com o espalhamento por fônons, é suficiente para explicar o mínimo na resistividade.

Entretanto, à medida que a temperatura tende a zero o termo logarítimo diverge, tornando evidente que o cálculo perturbativo a $T \rightarrow 0$ passa a não ser mais válido. Uma teoria válida nesse limite e que explica o comportamento do mínimo da resistividade ficou conhecida como o "Problema Kondo".

Usando técnicas de teorias de muito corpos e realizando o somatório sobre termos logarítimicos de diferentes ordens, no caso da interação antiferromagnética, chega-se a uma divergência na susceptibilidade magnética da impureza a uma temperatura fixa $T_{K}$, conhecida como temperatura Kondo, que dá conta da correlação antiferromagnética entre o spin da impureza localizada e os spin dos elétrons de condução (4). Ela pode ser escrita como

$$
k_{B} T_{K} \sim 2 D e^{-\frac{1}{2 J \rho_{0}}},
$$

onde $J$ é o termo de troca, $\rho_{0}$ é a densidade de estados de elétrons de condução no nível de Fermi e $D$ é a semi-largura de banda. 


\section{3}

\section{Pontos Quânticos}

Pontos quânticos (PQ) são estruturas capazes de confinar elétrons em pequenas regiões e, devido a suas dimensões nanoscópicas, um PQ pode apresentar tanto características de sistemas quânticos como de semicondutores eletrônicos. Nas últimas décadas, com o avanço das técnicas de litografia, tornou-se possível confinar em um PQ um número mínimo de elétrons acoplados por tunelamento quântico a elétrons deslocalizados fora do PQ. Este dispositivo tem as propriedades de um o transistor de um elétron (6).

Um transistor de um elétron constitue um átomo artificial devido ao confinamento dos elétrons em uma pequena região do espaço, apresentando comportamento de quantização da energia e permitindo alto controle do número de elétrons localizados no $\mathrm{PQ}$, similarmente ao que acontece em átomos reais. Esta característica confere ao PQ um sistema natural para o estudo do efeito Kondo, uma vez que isto não é acessível a sistemas convencionais em que uma impureza magnética está disposta sobre um meio metálico. No PQ podemos, por exemplo, alterar o número de elétrons e a diferença de energia entre os estados localizados e o nível de Fermi. Também é possível estudar um único elétron localizado ao invés de uma distribuição estatística (6).

A figura 1.3 apresenta um PQ de GaAs/AlGaAs com múltiplas portas metálicas, onde o gás bidimensional de elétrons é armadilhado entre duas camadas com propriedades eletrônicas diferentes. O eletrodo da direita e os eletrodos superior e inferior, ambos da esquerda, controlam a quantidade de elétrons no PQ. Na parte superior e na parte inferior da figura encontram-se reservatórios de elétrons. O eletrodo do meio na esquerda é utilizado para controlar a diferença de energia entre o estado local e o nível de Fermi do gás bidimensional. O potencial que regula esta diferença é chamado de potencial de porta.

O tamanho do PQ, figura 1.3, é de fundamental importância para a observação do efeito Kondo. Na região de confinamento, cerca de 50 elétrons podem ser aprisionados a baixas temperaturas, permitindo que o efeito Kondo se torne visível quando a temperatura for menor que a $T_{K}$ do sistema.

Ao aplicar um campo magnético, pelo efeito Zeeman, as energias dos elétrons confinados e a densidade de estados do gás de Fermi são deslocados 


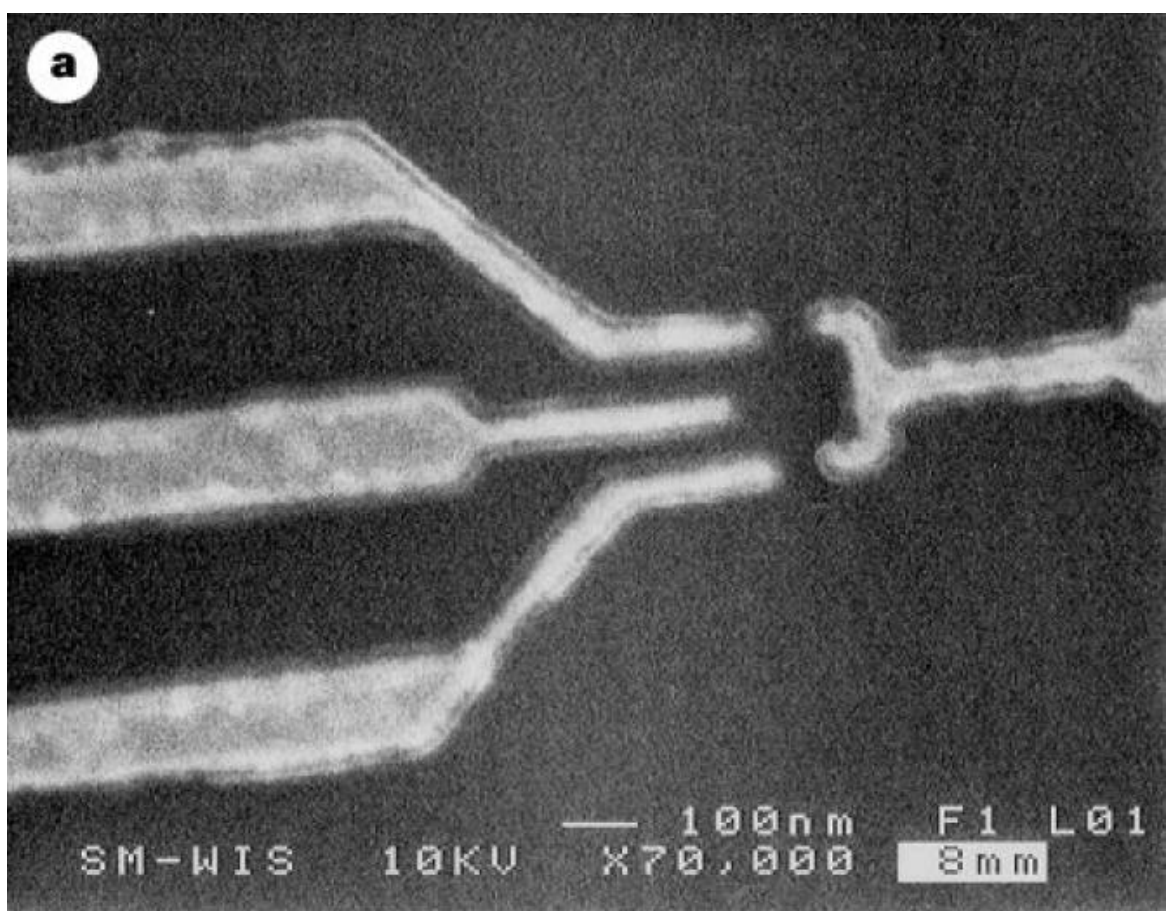

Figura 1.3: Imagem feita com escaneamento de microscopia eletrônica, (6).

por um fator $\mu_{B} B$ para cima ou para baixo, dependendo do spin eletrônico, onde $\mu_{B}$ é o magneton de Bohr e $B$ o módulo do campo magnético aplicado. Esta separação dos níveis de energia pode ser observada para temperaturas da ordem de mK e campo magnético suficientemente alto para separar os picos da condutância diferencial, figura 1.4, fornecendo uma assinatura característica do efeito Kondo. Quando a energia correspondente ao campo magnético $\mu_{B} B$ é maior que a temperatura Kondo $T_{K}$ o efeito Kondo é destruído, (6).

\section{4}

\section{Modelo de Anderson}

É interessante desenvolver modelos simples que descrevem a física de uma impureza magnética interagindo com elétrons de condução. Com este objetivo modelamos o problema utilizando o modelo de Anderson, o qual pode ser transformado no Hamiltoniano de Kondo usando uma transformação de Schrieffer-Wolff (7).

O modelo de Anderson permite explicar a existência de momentos magnéticos localizados, no meio de uma nuvem de elétrons deslocalizados. Ele inclui uma interação Coulombiana intereletrônica $U$ de curto alcance entre os 


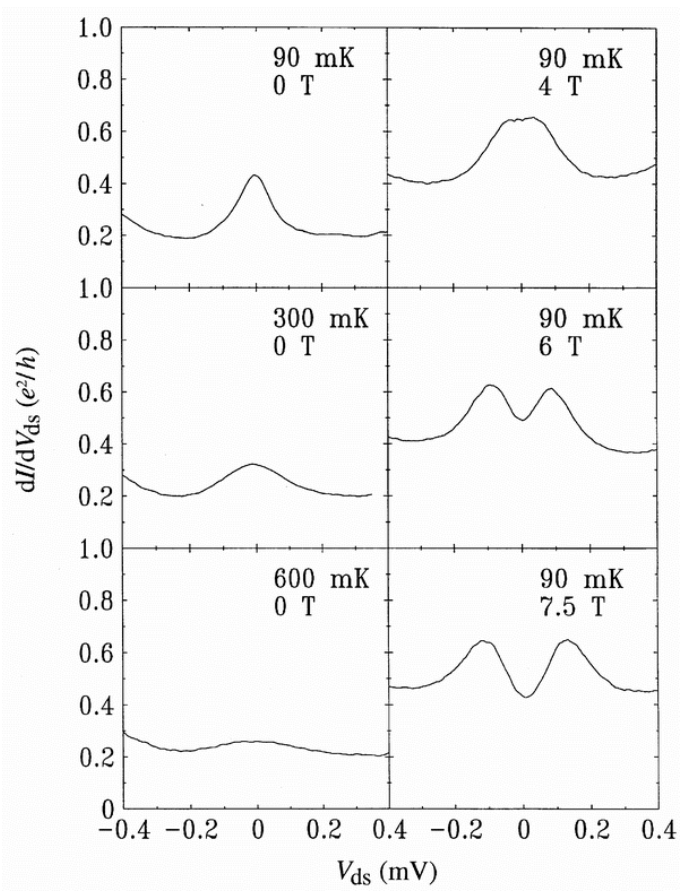

Figura 1.4: Condutância diferencial.

elétrons da impureza. Esta energia $U$ entre dois elétrons pertencentes a camada d ou f de um átomo é dada por

$$
U=\int \phi_{\mathrm{d}}^{*}(\mathbf{r}) \phi_{\mathrm{d}}^{*}\left(\mathbf{r}^{\prime}\right) \frac{e^{2}}{\left|\mathbf{r}-\mathbf{r}^{\prime}\right|} \phi_{\mathrm{d}}(\mathbf{r}) \phi_{\mathrm{d}}\left(\mathbf{r}^{\prime}\right) \mathrm{d} \mathbf{r} \mathrm{d} \mathbf{r}^{\prime},
$$

onde $\phi_{\mathrm{d}}$ é a função de onda eletrônica no orbital d da impureza, r e r' as posições dos elétrons e $e$ a carga eletrônica.

Como os elétrons estão no mesmo orbital atômico, pode existir uma significativa superposição das funções onda eletrônicas, de modo que a interação Coulombiana pode apresentar energias elevadas, da ordem de $30 \mathrm{eV}$. Para elétrons da camada 3d do íon da impureza em um meio hospedeiro metálico, é esperado uma redução nesse valor devido a deslocalização parcial da função de onda do elétron pelo meio hospedeiro. Estimativas tanto teóricas quanto experimentais revelam valores entre 1-7 eV, (4). O mesmo se aplica para impurezas de terras raras, visto que os níveis $4 \mathrm{f}$ são mais compactos e próximos ao núcleo atômico. No caso de $\mathrm{PQ}$, o valor de $U$ é muito inferior, da ordem de décimos de meV, dependendo de seu tamanho.

Na presença de um campo magnético externo o Hamiltoniano do modelo de Anderson apresenta a forma 


$$
H=\sum_{\sigma} \epsilon_{0 \sigma} n_{0 \sigma}+U n_{0 \sigma} n_{0 \bar{\sigma}}+\sum_{\mathbf{k}, \sigma} \epsilon_{\mathbf{k} \sigma} n_{\mathbf{k} \sigma}+\sum_{\mathbf{k}, \sigma} V_{\mathbf{k}}\left(d_{0 \sigma} c_{\mathbf{k} \sigma}^{\dagger}+d_{0 \sigma}^{\dagger} c_{\mathbf{k} \sigma}\right) .
$$

O primeiro termo do Hamiltoniano da equação (1.4.2) corresponde à energia do elétron localizado na impureza $\epsilon_{0 \sigma}$, onde o índice 0 refere-se a impureza e o índice $\sigma$ ao spin, que incorpora o efeito do campo magnético

$\operatorname{com} \sigma= \pm \frac{1}{2}$

$$
\epsilon_{0 \sigma}=\epsilon_{0}-\sigma \mu_{B} B
$$

O segundo termo corresponde à interação elétron-elétron de magnitude $U$, ambos localizados na impureza, e $n_{0 \sigma}$ ao operador número de partículas, com spin $\sigma$.

O terceiro corresponde à energia $\epsilon_{\mathbf{k} \sigma}$ do elétron de momento $\mathbf{k}$ e campo magnético externo $B$

$$
\epsilon_{\mathbf{k} \sigma}=\epsilon_{\mathbf{k}}-\sigma \mu_{B} B
$$

$n_{\mathbf{k} \sigma}$ é o operador número de elétrons com vetor de onda $\mathbf{k}$ e spin $\sigma$, em uma representação diagonal dos elétrons do cristal.

O quarto denota a interação entre a impureza e os elétrons de condução, sendo $V_{\mathbf{k}}$ o elemento de matriz que liga os estados do elétron de momento $\mathbf{k}$ no cristal com o elétron na impureza. O último termo estabelece a conexão entre a impureza e o cristal, onde $d_{0 \sigma}$ é o operador de aniquilação de elétrons na impureza, $c_{\mathbf{k} \sigma}^{\dagger}$ é o operador de criação do elétron no cristal, $d_{0 \sigma}^{\dagger}$ é o operador de criação de um elétron na impureza e $c_{\mathbf{k} \sigma}$ é o operador de aniquilação de um elétron no cristal.

É interessante e de fundamental importância estudar o comportamento da energia $E=\langle H\rangle$ como função da energia da impureza, já que sua derivada com respeito a $e_{0}$ corresponde ao estado de carga na impureza do sistema

$$
\frac{\partial}{\partial e_{0}} E=\sum_{\sigma}\left\langle n_{0 \sigma}\right\rangle=\left\langle n_{0}\right\rangle \text {. }
$$

O número de ocupação de elétrons na impureza $n_{0}$ nos permite identificar se o sistema encontra-se no regime de flutuação de carga ou no regime Kondo, em que o $\left\langle n_{0}\right\rangle \approx 1$.

O campo magnético externo tem por finalidade produzir uma perturbação no elétron localizado na impureza, originando uma magnetização M 
do sistema.

A auto-energia do sistema $E$ possuiu uma dependência com o campo magnético $B$ dada por

$$
E \sim\left(\left\langle n_{0 \sigma}-n_{0 \bar{\sigma}}\right\rangle+\sum_{\mathbf{k}}\left\langle n_{\mathbf{k} \sigma}-n_{\mathbf{k} \bar{\sigma}}\right\rangle\right) \mu_{B} B .
$$

De modo que ao derivarmos a energia com respeito ao campo magnético obtemos a magnetização do sistema

$$
M=-\frac{\partial}{\partial B} E
$$

Por sua vez, a susceptibilidade magnética do sistema $\chi$ é obtida através da derivada segunda da auto-energia do sistema

$$
\chi=\frac{\partial^{2}}{\partial B^{2}} E .
$$

O estado fundamental pode ser estudado por métodos não perturbativos como o método variacional. A função de onda mais simples de spin meio utilizada no método variacional é a de um singleto, que no caso antiferromagnético é utilizada como ansatz para o cálculo da susceptibilidade magnética do estado fundamental de uma banda plana, obtendo como resultado (4)

$$
\chi=\frac{\left(g \mu_{B}\right)^{2}}{4 k_{B} T_{K}},
$$

onde $g$ é o fator de Landé. Assim, podemos obter a dependência da temperatura Kondo do sistema com a susceptibilidade magnética

$$
T_{K} \propto \frac{1}{\chi}
$$

que pode ser calculada para diferentes valores dos parâmetros do sistema.

No próximo capítulo discutiremos um formalismo baseado em operadores de projeção para o cálculo da energia do estado fundamental, principal objetivo do presente trabalho, permitindo o cálculo da magnetização, da susceptibilidade magnética e da temperatura Kondo do sistema. 


\section{2 \\ Determinação da energia do Hamiltoniano de Anderson utilizando um formalismo de operadores de projeção}

\section{1 \\ Introdução}

O Hamiltoniano da Impureza de Anderson recebeu muita atenção nos últimos 50 anos, desde o trabalho pioneiro de Kondo que explicou o comportamento da resistividade como função da temperatura de metais dopados com impurezas magnéticas, sendo, este Hamiltoniano, a base da física de férmions pesados. Como um Hamiltoniano de Hubbard de infinitas dimensões pode ser transformado no Hamiltoniano da Impureza de Anderson através de uma renormalização autoconsistente, usando Dynamical Mean Field Theory (DMFT) (8), esse modelo permitiu o estudo de muitos aspectos da física de muitos corpos, como por exemplo a transição metal isolante. Mais recentemente o efeito Kondo recebeu grande atenção associada com as propriedades de muitos corpos de sistemas eletrônicos nanoscópicos fortemente correlacionados, como pontos quânticos (PQ) ou estruturas de pontos quânticos.

O Hamiltoniano da Impureza de Anderson foi resolvido por uma variedade de abordagens numéricas diferentes, como Logarithmic Discretization Embedded Cluster Approximation (LDECA) (9), Numerical Renormalization Group (NRG) (10) e Density Matrix Renormalization Group (DMRG) (11) ou métodos mais algébricos como Slave Bosons (12) e Non-Crossing Approximations (NCA) (13), (14), One-Crossing Approximations (OCA) (15), Monte Carlo Quântico (16), Equações de Movimento (17) e Bethe Ansatz (18). Apesar dos dois primeiros métodos poderem ser considerados exatos, enquanto os outros são aproximações com diferentes capacidades de descrever a física envolvida, eles possuem muitas limitações em relação à complexidade dos sistemas e a temperatura. Além disso, as propriedades dinâmicas não são completamente bem caracterizadas por métodos numéricos. 
Proporemos neste capítulo um formalismo algébrico baseado em operadores de projeção para calcular a enegia total do sistema em função dos parâmetros que o definem. O formalismo baseia-se em projetar o espaço de Hilbert em um subespaço onde os elétrons estão preenchendo o mar de Fermi até o nível de Fermi. Para operar neste subespaço o Hamiltoniano tem que ser renormalizado através de um processo sistemático e autoconsistente, o qual será apresentado durante este capítulo e permite obter as propriedades do estado fundamental. Conhecendo a energia do sistema $E=\langle H\rangle$ para diferentes valores do campo magnético $B$ é possível determinar a magnetização e a susceptibilidade magnética do mesmo, além de obter a temperatura Kondo como função dos parâmetros do sistema. Podemos, ainda, determinar a ocupação eletrônica média na impureza para diferentes valores da energia da impureza $e_{0 \sigma}$ e também é possível obter a condutância em função dos parâmetros do sistema aplicando a regra da soma de Friedel (4), (19).

\section{2}

\section{Decompondo o espaço de Hilbert}

Com o objetivo de obter a energia do estado fundamental do sistema físico, vamos decompor o espaço de Hilbert em dois subespaços, os quais denotaremos por $|1\rangle$ e $|2\rangle$, utilizando operadores de projeção $P_{1}$ e $P_{2}$, onde o operador $P_{1}$ projeta o espaço de Hilbert no subespaço $|1\rangle$,

$$
P_{1}=|1\rangle\langle 1|
$$

e o operador $P_{2}$ no subespaço $|2\rangle$,

$$
P_{2}=|2\rangle\langle 2| .
$$

Desde que

$$
P_{1}+P_{2}=\mathbf{1}
$$

todo operador definido no espaço de Hilbert pode ser representado por combinações lineares de $|1\rangle$ e $|2\rangle$, figura 2.1 .

Aplicando o operador $P_{1}+P_{2}$, que é uma identidade, em ambos os lados de um Hamiltoniano geral $H$ temos,

$$
H=\left(P_{1}+P_{2}\right) H\left(P_{1}+P_{2}\right)=H_{11}+H_{12}+H_{21}+H_{22},
$$

onde $H_{i j} \equiv P_{i} H P_{j}=|i\rangle\langle i|H| j\rangle\langle j|$.

Por outro lado, um auto-estado da equação de Schrödinger com energia 


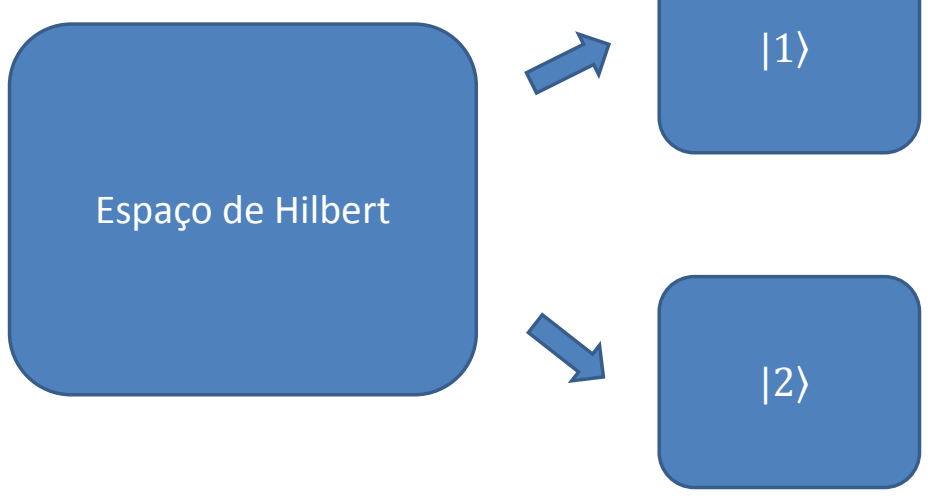

Figura 2.1: Decomposição do espaço de Hilbert em dois subespaços, $|1\rangle$ e $|2\rangle$.

$E$ apresenta a forma

$$
H|\psi\rangle=E|\psi\rangle
$$

com $|\psi\rangle$ sendo um vertor pertencente ao espaço de Hilbert.

Substituindo a equação (2.2.4) na equação (2.2.5) temos

$$
\left(H_{11}+H_{12}+H_{21}+H_{22}\right)\left(c_{1}|1\rangle+c_{2}|2\rangle\right)=E\left(c_{1}|1\rangle+c_{2}|2\rangle\right),
$$

onde escrevemos $|\psi\rangle$ como uma combinação linear de vetores nos subespaços $|1\rangle$ e $|2\rangle$ e $c_{1}$ e $c_{2}$ são constantes.

Na equação (2.2.6), os termos do tipo $H_{i j}|i\rangle=|i\rangle\langle i|H| j\rangle\langle j \mid i\rangle$ são nulos, só sobrevivendo os termos do tipo $H_{i j}|j\rangle=|i\rangle\langle i|H| j\rangle\langle j \mid j\rangle$, o que nos permite escrever a equação (2.2.6) como duas equações e estabeler uma relação entre os subespaços $|1\rangle$ e $|2\rangle$

$$
\begin{gathered}
H_{11}|1\rangle+H_{12}|2\rangle=E|1\rangle \\
H_{21}|1\rangle+H_{22}|2\rangle=E|2\rangle,
\end{gathered}
$$


obtendo, assim,

$$
\begin{gathered}
|1\rangle=\left(E-H_{11}\right)^{-1} H_{12}|2\rangle \\
|2\rangle=\left(E-H_{22}\right)^{-1} H_{21}|1\rangle .
\end{gathered}
$$

Substituindo a equação (2.2.8) na equação (2.2.7) temos

$$
H_{11}|1\rangle+H_{12}\left(E-H_{22}\right)^{-1} H_{21}|1\rangle=E|1\rangle .
$$

A equação (2.2.9) é uma nova equação com os mesmos autovalores da equação de Schrödinger do Hamiltoniano original atuando somente no subespaço projetado $|1\rangle$, que poderá ser um subespaço muito simples, por exemplo, de uma única autofunção como mostraremos mais adiante. A equação de Schrödinger do Hamiltoniano de Anderson é mapeada, desta forma, em uma nova equação com os autovalores originais preservados. A dificuldade a ser resolvida está agora na obtenção do Hamiltoniano renormalizado

$$
H_{r} \equiv H_{11}+H_{12}\left(E-H_{22}\right)^{-1} H_{21},
$$

o qual terá que ser resolvido autoconsistentemente para cada valor da energia $E$ do sistema.

\section{3}

\section{Construção dos subespaços}

Até o presente momento não estabelecemos nenhuma condição sobre os subespaços supracitados, bem como sobre operadores de projeções $P_{1}$ e $P_{2}$, de modo que as relações obtidas são absolutamente gerais. Entretanto, se escolhermos de forma conveniente esses subespaços poderemos facilitar o cálculo da energia $E$ de um auto-estado do Hamiltoniano, o que implica a solução autoconsistente da equação (2.2.9).

Para a obtenção da energia do estado fundamental do Hamiltoniano de Anderson (equação (1.4.2) do capítulo anterior)

$$
H=\sum_{\sigma} \epsilon_{0 \sigma} n_{0 \sigma}+U n_{0 \sigma} n_{0 \bar{\sigma}}+\sum_{\mathbf{k}, \sigma} \epsilon_{\mathbf{k} \sigma} n_{\mathbf{k} \sigma}+\sum_{\mathbf{k}, \sigma} V_{\mathbf{k}}\left(d_{0 \sigma} c_{\mathbf{k} \sigma}^{\dagger}+d_{0 \sigma}^{\dagger} c_{\mathbf{k} \sigma}\right),
$$

onde estamos acrescentando os termos da aplicação de um campo magnético externo, visto que vamos estar interessados no cálculo da susceptibilidade magnética do sistema, escolheremos como subespaço $|1\rangle$ o estado da impureza desocupada e o mar de Fermi com todos os estado ocupados até o nível de 
Fermi, conforme o diagrama da figura 2.2.

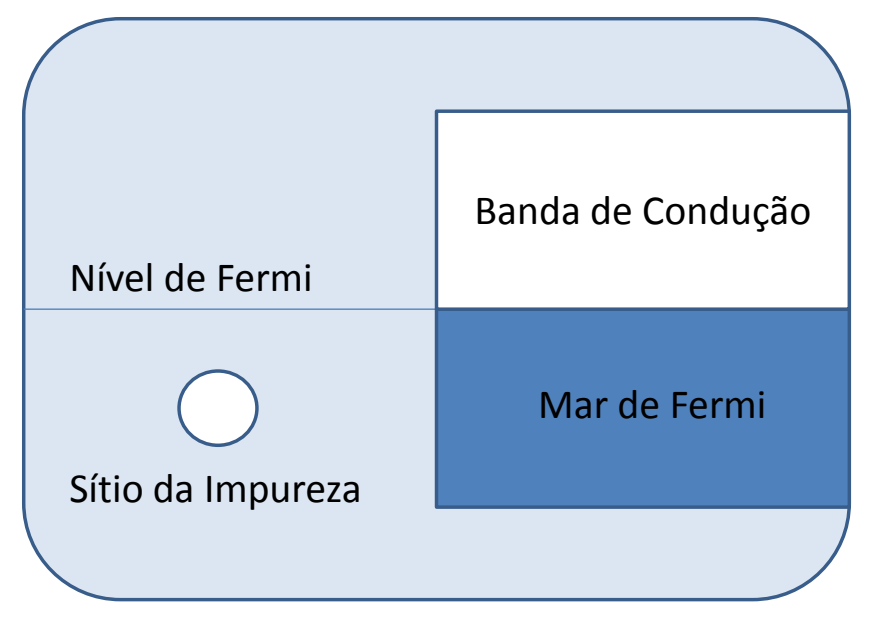

Figura 2.2: Escolha do subespaço $|1\rangle$ como sendo o do mar de Fermi cheio e a impureza e a banda de condução vazias.

O operador $H_{11}$, será dado pelo somatório do operador número de elétrons $n_{\mathbf{k} \sigma}$ presentes no estado fundamental do mar de Fermi de $N$ elétrons, cada um com sua energia $\epsilon_{\mathbf{k}}$, onde o índice $\mathbf{k}$ é o vetor número de onda e $\sigma$ o spin de cada elétron; fica implícito que tomamos o mar de Fermi numa representação já diagonalizada,

$$
H_{11}=\sum_{\epsilon_{\mathbf{k} \sigma}<\epsilon_{F}} \epsilon_{\mathbf{k} \sigma} n_{\mathbf{k} \sigma}
$$

onde $\epsilon_{F}$ é a energia de Fermi . O operador de projeção $P_{1}$ possui a forma,

$$
P_{1}=\prod_{\epsilon_{\mathbf{k} \sigma}<\epsilon_{F}} n_{\mathbf{k} \sigma}
$$

Usando o princípio da exclusão de Pauli, o valor esperado do operador $H$ no subespaço $|1\rangle$ é calculado pelo somatório da energia de cada elétron no mar de Fermi

$$
h_{11} \equiv\langle 1|H| 1\rangle=\epsilon_{T}=\sum_{\epsilon_{\mathbf{k} \sigma}<\epsilon_{F}} \epsilon_{\mathbf{k} \sigma}
$$

O subespaço $|2\rangle$ será constituído de todo estado não pertencente ao subespaço $|1\rangle$. O operador de projeção $P_{2}$ pode ser escrito como,

$$
P_{2}=1-\prod_{\epsilon_{\mathbf{k} \sigma}<\epsilon_{F}} n_{\mathbf{k} \sigma}
$$


As funções que compõem este subespaço podem ser obitidas através de sucessivas aplicações do Hamiltoniano $H_{21}$, que conecta o subespaço $|1\rangle$ ao $|2\rangle$, como será mostrado adiante.

Ao observar-se atentamente o Hamiltoniano de Anderson notamos que o termo responsável pela conexão entre os subespaços $|1\rangle$ e $|2\rangle(|2\rangle$ e $|1\rangle)$ é o próprio termo de hibridização, que aniquila (cria) um elétron no mar de Fermi e cria (aniquila) um elétron na impureza

$$
\begin{aligned}
& H_{21}=\sum_{\mathbf{k}, \sigma} V_{\mathbf{k}}\left(d_{0 \sigma}^{\dagger} c_{\mathbf{k} \sigma}\right), \\
& H_{12}=\sum_{\mathbf{k}, \sigma} V_{\mathbf{k}}\left(d_{0 \sigma} c_{\mathbf{k} \sigma}^{\dagger}\right) .
\end{aligned}
$$

Onde $d_{0 \sigma}^{\dagger}\left(d_{0 \sigma}\right)$ é o operador de criação (aniquilação) de um elétron na impureza com spin $\sigma$ e $c_{\mathbf{k} \sigma}^{\dagger}\left(c_{\mathbf{k} \sigma}\right)$ é o operador de criação (aniquilaçao) de um elétron no mar de Fermi com spin $\sigma$.

Partiremos do mar de Fermi com $N$ elétrons no seu estado fundamental, subespaço $|1\rangle$. Ao aplicarmos o operador $H_{21}$ ao subespaço $|1\rangle$ criamos um elétron localizado na impureza e um buraco no mar de Fermi. A esta função do subespaço $|2\rangle$ chamaremos de estado I. A cada aplicação dos operadores $H_{12}$ e $H_{21}$ teremos a possibilidade de gerar um novo estado, originando o estado I, o estado II e assim por diante, todos pertencentes ao subespaço $|2\rangle$.

Consideramos a interação intereletrônica na impureza $U$ muito forte, pois estamos nos concentrando em impurezas de elementos químicos pertencentes tipicamente aos terras raras ou metais de transição, em que a localização das funções de onda que descreve os elétrons nas camadas $3 \mathrm{~d}$ ou $4 \mathrm{f}$ origina uma forte repulsão Coulombiana. Tomaremos o limite em que $U \rightarrow \infty$, de modo que estados de dupla ocupação na impureza não estarão no espaço de Hilbert em que iremos trabalhar.

Como $U \rightarrow \infty$, partindo do estado I, antes de criar um outro elétron na impureza devemos levar o elétron já presente à banda de condução. Aplicando o operador $H_{12}$ destruímos o elétron na impureza e o criamos no estado da banda de condução, até então vazia, denotado pelo vetor número de onda $\mathbf{K}$ e com energia $\epsilon_{\mathbf{K}}$, para todo $\epsilon_{\mathbf{K}}>\epsilon_{F}$. Este é o estado II.

Contudo, ao aplicarmos o operador $H_{12}$ poderíamos fazer com que o 
elétron localizado na impureza ocupasse o buraco existente no mar de Fermi, todavia para que este elétron ocupe o buraco, ele deve possuir um vetor número de onda $\mathbf{k}$ específico igual ao do buraco, frente a todos os valores de $\mathbf{k}$ possíveis representados pelo somatório na equação (2.3.7). Como ficará mais evidente adiante, este termo de possuir um $\mathbf{k}$ específico não envolve um somatório e portanto não contribui ao cálculo.

Com a impureza vazia, no estado II, podemos aplicar novamente o operador $H_{12}$ e continuar o processo indefinidamente, como no diagrama ilustrado na figura 2.3. Este processo não esvaziará o mar de Fermi pois no limite termodinâmico o mar de Fermi tende a um contínuo.

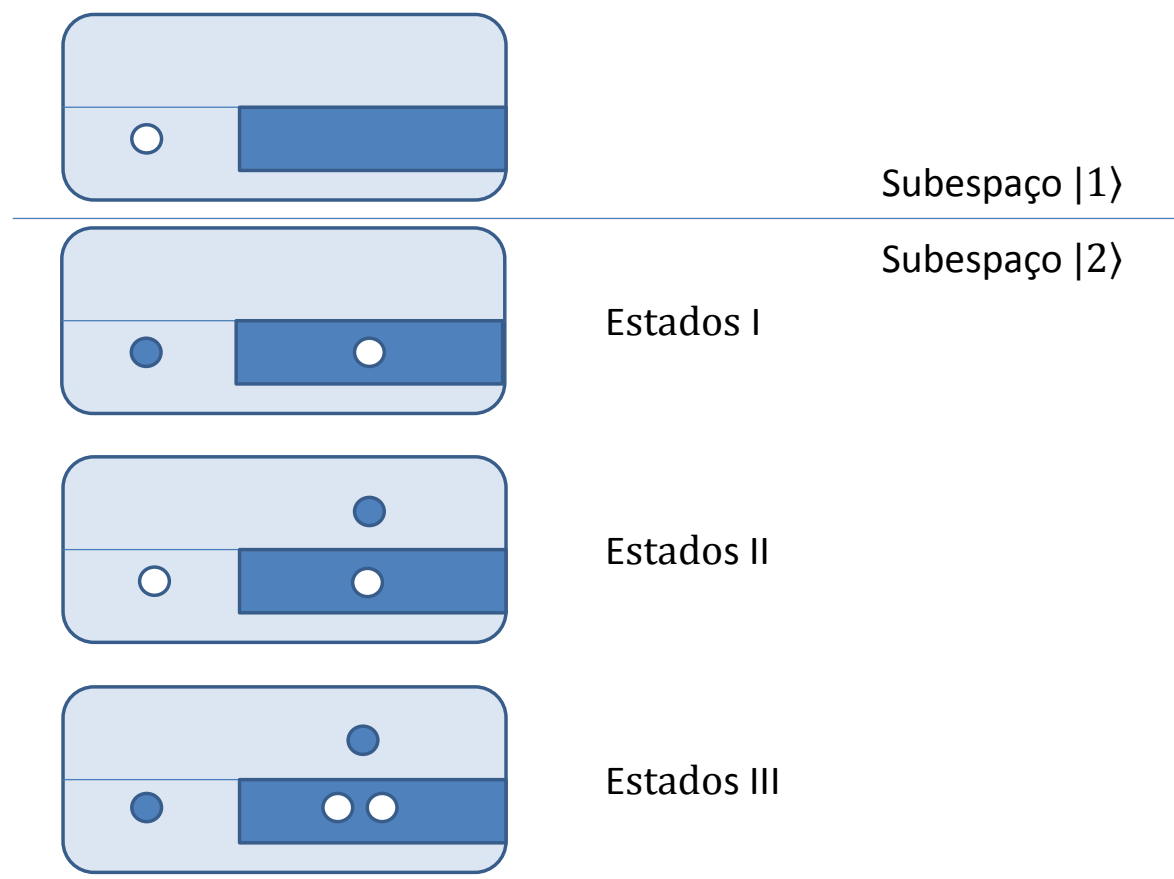

Figura 2.3: Construção do subespaço $|2\rangle$, representando esquematicamente três famílias de estados.

Observando o diagrama da figura 2.3 e pelo discutido acima, nota-se que o estado I é análogo ao estado III a menos de um buraco no mar de Fermi e um elétron na banda de condução acima do nível de Fermi, pois ambos os estados consistem em criar um elétron na impureza e aniquilar um elétron no mar de Fermi. Esta característica corrobora a ideia de que, no limite termodinâmico, quando o número de estados disponíveis e de partículas tende a infinito o problema admite uma solução autoconsistente. 


\section{4}

\section{Cálculo da correção da energia}

Todavia, ainda não calculamos a energia, apresentamos somente a ideia do formalismo proposto para encontrá-la. Nesta secção partiremos para o cálculo da energia propriamente dito.

Como o pricipal objetivo é propor um novo método para a solução do Hamiltoniano de Anderson, supomos que

$$
V_{\mathbf{k}}=\frac{V}{\sqrt{N}} \quad \forall \mathbf{k},
$$

ou melhor, que o valor da hibridização independa de $\mathbf{k}$ e seja uma constante igual a $V$, onde devemos escalar com um fator $\frac{1}{\sqrt{N}}$, sendo $N$ o número de estados no mar de Fermi, para preservar o valor finito das interações.

O cálculo terá como base a equação (2.2.9), onde temos a energia do subespaço $|1\rangle$, equação (2.3.4), devemos inverter o operador $\left(E-H_{22}\right)$ representado no subespaço $|2\rangle$ e encontrar o elemento de matriz imediatamente contíguo ao subespaço $|1\rangle$ dado por $H_{12}\left(E-H_{22}\right)^{-1} H_{21}$.

Inicialmente calcularemos os elementos de matriz do operador $H_{22}$ levando-se em consideração o estado que resulta da aplicação do $H_{21}$ sobre do estado I, resultando

$$
h_{22}^{(1)}=\epsilon_{T}-\epsilon_{\mathbf{k} \sigma}+\epsilon_{0 \sigma},
$$

onde $\epsilon_{\mathbf{k} \sigma}$ é a a energia do elétron destruído com $\epsilon_{\mathbf{k} \sigma}<\epsilon_{F}$ e $\epsilon_{0 \sigma}$ a energia do elétron criado na impureza.

Como discutido no capítulo anterior, a energia do elétron retirado do mar de Fermi $\epsilon_{\mathbf{k} \sigma}$ é caracterizada por um vetor número de onda $\mathbf{k}$, ao passo que a energia do elétron na impureza apresenta um subíndice $\sigma$ devido ao campo magnético externo, onde o spin do elétron $\sigma$ pode-se alinhar paralelamente ou antiparalelamente ao campo, ou seja,

$$
\epsilon_{0 \sigma}=\epsilon_{0} \pm \frac{B}{2}
$$

onde $\epsilon_{0}$ é a energia do elétron na impureza a campo nulo e $B$ o módulo do campo magnético externo.

Sendo assim, calculamos a energia com a renormalização que surge 
de inverter o operador $\left(E-H_{22}\right)$ considerando unicamente as contribuições que provém do estado I. Devemos somar em $\mathbf{k}$ para incorporar todas as contribuições de todos os elétrons destruídos por baixo do nível de Fermi e o somatório em $\sigma$ correspondem aos valores do spin.

$$
\epsilon_{T}+\frac{1}{N} \sum_{\epsilon_{\mathbf{k} \sigma<\epsilon_{F}}} \frac{V^{2}}{E-\epsilon_{T}+\epsilon_{\mathbf{k} \sigma}-\epsilon_{0 \sigma}-\ldots}=E
$$

Observe que para calcular a energia do estado I devemos conhecer a energia do estado II. O elemento de matriz $h_{22}^{(2)}$ referente ao estado II pode ser escrito,

$$
h_{22}^{(2)}=\epsilon_{T}-\epsilon_{\mathbf{k} \sigma}+\epsilon_{\mathbf{K} \sigma},
$$

onde $\epsilon_{\mathbf{K} \sigma}$ é a energia do elétron ocupando o estado de momento $\mathbf{K}$ acima do nível de Fermi.

Seguindo adiante, levaremos em conta a correção na energia do estado I devido ao estado II. Invertendo o operador $E-H_{22}$ e incluindo o estado II termos,

$$
\epsilon_{T}+\frac{1}{N} \sum_{\epsilon_{\mathbf{k} \sigma}<\epsilon_{F}} \frac{V^{2}}{E-\epsilon_{T}+\epsilon_{\mathbf{k} \sigma}-\epsilon_{0 \sigma}-\frac{1}{N} \sum_{\epsilon_{\mathbf{K} \sigma}>\epsilon_{F}} \frac{V^{2}}{E-\epsilon_{T}+\epsilon_{\mathbf{k} \sigma}-\epsilon_{\mathbf{K} \sigma}-\ldots}}=E .
$$

Continuando o mesmo processo, podemos escrever

$$
h_{22}^{(3)}=\epsilon_{T}-\epsilon_{\mathbf{k} \sigma}+\epsilon_{\mathbf{K} \sigma}-\epsilon_{\mathbf{k}^{\prime} \sigma}+\epsilon_{0 \sigma},
$$

onde $\epsilon_{\mathbf{k}}{ }^{\prime} \sigma$ é a energia do segundo elétron destruído, com vetor número de onda que em princípio deve satisfazer $\mathbf{k}^{\prime} \neq \mathbf{k}$, pois o elétron $\mathbf{k}$ já foi aniquilado no estado anterior

Com a correção do estado III temos:

$$
\begin{array}{r}
\epsilon_{T}+\frac{1}{N} \sum_{\epsilon_{\mathbf{k} \sigma}<\epsilon_{F}} \frac{V^{2}}{E-\epsilon_{T}+\epsilon_{\mathbf{k} \sigma}-\epsilon_{0 \sigma}-\frac{1}{N} \sum_{\epsilon_{\mathbf{K} \sigma}>\epsilon_{F}} \frac{V^{2}}{E-\epsilon_{T}+\epsilon_{\mathbf{k} \sigma}-\epsilon_{\mathbf{K} \sigma}-\Sigma}}=E \\
\Sigma=\frac{1}{N} \sum_{\epsilon_{\mathbf{k}} \sigma<\epsilon_{F}} \frac{V^{2}}{E-\epsilon_{T}+\epsilon_{\mathbf{k} \sigma}-\epsilon_{\mathbf{K} \sigma}+\epsilon_{\mathbf{k}}{ }^{\prime}-\epsilon_{0 \sigma}-\ldots} .
\end{array}
$$

Note que a renormalização da energia devido ao estado III é muito 
semelhante a renormalização devido ao estado I, o que já era esperado. Vemos também, que começa a surgir uma simetria de recorrência que podemos identificar. Considerando renormalizações de estados de ordem superior no limite termodinâmico quando $N$ tende a infinito, teremos uma estrutura de fração continuada infinita, o que permite um cálculo autoconsistente.

É conveniente definir as funções

$$
\begin{aligned}
f_{1 \sigma}(\xi) & \equiv \frac{1}{N} \sum_{\epsilon_{\mathbf{k} \sigma}<\epsilon_{F}} \frac{V^{2}}{\xi+\epsilon_{\mathbf{k} \sigma}-\epsilon_{0 \sigma}-f_{2 \sigma}\left(\xi+\epsilon_{\mathbf{k} \sigma}\right)} \\
f_{2 \sigma}(\xi) & \equiv \frac{1}{N} \sum_{\epsilon_{\mathbf{K} \sigma}>\epsilon_{F}} \frac{V^{2}}{\xi-\epsilon_{\mathbf{K} \sigma}-\sum_{\sigma} f_{1 \sigma}\left(\xi-\epsilon_{\mathbf{K} \sigma}\right)}
\end{aligned}
$$

e $\Delta E \equiv E-\epsilon_{T}$, de modo que o equivalente da equação (2.4.8) para renormalizações de infinitos estágios escreve-se como

$$
\Delta E=\sum_{\sigma} f_{1 \sigma}(\Delta E)
$$

para $\sigma \neq \bar{\sigma}$. A determinação da função $f_{1 \sigma}(\xi)$ permite em princípio calcular os autovalores $E$ do sistema.

\section{5}

\section{Passagem ao contínuo}

No limite termodinâmico em que $N \rightarrow \infty$ podemos eliminar a restrição $\mathbf{k} \neq \mathbf{k}$ ', visto que a diferença entre eliminar, e não eliminar o termo de $\mathbf{k}$ no somatório em $\epsilon_{\mathbf{k}}$ ' ${ }$ tende a zero neste limite. Também neste limite, os somatórios em $\epsilon_{\mathbf{k} \sigma}$ podem ser escritos como

$$
\frac{1}{N} \sum_{\epsilon_{\mathbf{k} \sigma}} f\left(\epsilon_{\mathbf{k} \sigma}\right)=\int \frac{1}{N} \sum_{\epsilon_{\mathbf{k} \sigma}} \delta\left(x-\epsilon_{\mathbf{k} \sigma}\right) f(x) d x,
$$

onde $\delta\left(x-x^{\prime}\right)$ é a função delta de Dirac. Com isso, a equação (2.4.9) pode ser escrita como

$$
\begin{aligned}
f_{1 \sigma}(\xi) & \equiv V^{2} \int_{-\infty}^{\epsilon_{F}} \frac{\rho_{\sigma}(x)}{\xi+x-\epsilon_{0 \sigma}-f_{2 \sigma}(\xi+x)} d x \\
f_{2 \sigma}(\xi) & \equiv V^{2} \int_{\epsilon_{F}}^{\infty} \frac{\rho_{\sigma}(x)}{\xi-x-\sum_{\sigma} f_{1 \sigma}(\xi-x)} d x
\end{aligned}
$$


com $\rho_{\sigma}(x)$ sendo a densidade de estados da banda de condução do sistema, dada por

$$
\rho_{\sigma}(x) \equiv \frac{1}{N} \sum_{\epsilon_{\mathbf{k} \sigma}} \delta\left(x-\epsilon_{\mathbf{k} \sigma}\right) .
$$

Nesse limite, as renormalizações da energia nos estados I e III na equação (2.4.8) passam a ser iguais, assim, o problema consiste em resolver as equações (2.5.2) e (2.4.10), as quais implicam numa solução autoconsistente. Entretanto, antes de buscarmos a solução é interessante notar algumas propriedades das equações a serem resolvidas.

Para conhecer a função $f_{1 \sigma}(\xi)$ precisamos realizar uma integração e, portanto, conhecer a função $f_{2 \sigma}(\xi)$, ao passo que para conhecer $f_{2 \sigma}(\xi)$, precisamos conhecer $f_{1 \sigma}(\xi)$, o que define o processo autoconsistente. O fato da expressão (2.5.2) a ser resolvida implique no cálculo de uma integral, transforma a solução autoconsistente num problema não local.

Os pólos das integrais também não são bem conhecidos, apesar de ter um vínculo simples que define a localização dos pólos. Basta olhar para o denominador das funções para perceber que não podemos determinar sua localização pois não conhecemos as funções $f_{1 \sigma}(\xi)$ e $f_{2 \sigma}(\xi)$, bem como pelo fato das funções estarem deslocadas pela variável de integração.

Não obstante, as equações (2.5.2) são similares a uma transformada de Hilbert, de modo que esperamos que certas propriedades da transformada de Hilbert sejam encontradas na solução. Em especial esperamos que um pico em uma das funções introduza uma oscilação na outra função, como nas relaçoes de Kramers-Kronig, tão conhecidas do eletromagnetismo e do formalismo das funções de Green.

Realizando uma mudança de variáveis

$$
\begin{array}{ccc}
y \equiv \xi+x & \text { para } & f_{1 \sigma}(\xi) \\
y \equiv \xi-x & \text { para } & f_{2 \sigma}(\xi)
\end{array}
$$


Um formalismo de operadores de projeção para resolver o Hamiltoniano de Anderson

podemos escrever $f_{1 \sigma}(\xi)$ e $f_{2 \sigma}(\xi)$ como

$$
\begin{aligned}
f_{1 \sigma}(\xi) & =V^{2} \int_{-\infty}^{\xi+\epsilon_{F}} \frac{\rho_{\sigma}(y-\xi)}{y-\epsilon_{0 \sigma}-f_{2 \sigma}(y)} d y \\
f_{2 \sigma}(\xi) & =V^{2} \int_{-\infty}^{\xi-\epsilon_{F}} \frac{\rho_{\sigma}(\xi-y)}{y-\sum_{\sigma} f_{1 \sigma}(y)} d y
\end{aligned}
$$

forma que será útil mais adiante.

\section{6}

\section{Banda plana e o comportamento da derivada}

A forma mais simples de representar a banda de condução é a de uma banda plana, que se estende de $-D$ a $D$ para o caso em que não há campo magnético externo. Fazendo esta suposição a equação (2.5.3) fica

$$
\rho_{\sigma}(x)=\left\{\begin{array}{lll}
\frac{1}{2 D}, & \text { para } & |x|<D \\
0, & \text { para } & |x|>D
\end{array} .\right.
$$

Para a banda plana, as expressões para $f_{1 \sigma}(\xi)$ e $f_{2 \sigma}(\xi)$, equações (2.5.2), ficam

$$
\begin{aligned}
f_{1 \sigma}(\xi) & =\frac{V^{2}}{2 D} \int_{-D-\sigma \frac{B}{2}}^{\epsilon_{F}} \frac{1}{\xi+x-\epsilon_{0 \sigma}-f_{2 \sigma}(\xi+x)} d x \\
f_{2 \sigma}(\xi) & =\frac{V^{2}}{2 D} \int_{\epsilon_{F}}^{D-\sigma \frac{B}{2}} \frac{1}{\xi-x-\sum_{\sigma} f_{1 \sigma}(\xi-x)} d x
\end{aligned}
$$

ou usando as transformações da equação (2.5.4), ficam

$$
\begin{aligned}
f_{1 \sigma}(\xi) & =\frac{V^{2}}{2 D} \int_{\xi-D-\sigma \frac{B}{2}}^{\xi+\epsilon_{F}} \frac{1}{y-\epsilon_{0 \sigma}-f_{2 \sigma}(y)} d y \\
f_{2 \sigma}(\xi) & =\frac{V^{2}}{2 D} \int_{\xi-D+\sigma \frac{B}{2}}^{\xi-\epsilon_{F}} \frac{1}{y-\sum_{\sigma} f_{1 \sigma}(y)} d y
\end{aligned}
$$

onde a presença do campo magnético externo, em unidades de energia, é levada em consideração nos limites de integração das equações (2.6.2) e (2.6.3).

É interessante calcular as derivadas de $f_{1 \sigma}(\xi)$ e $f_{2 \sigma}(\xi)$ para uma banda 
plana, entretanto, nos restringiremos ao caso sem campo magnético externo sem perda de generalidade. Para tal, teremos de calcular derivadas do tipo

$$
\frac{d}{d x}\left(\int_{h(x)}^{g(x)} f(t) d t\right)
$$

a qual pode ser calculada com a regra da cadeia. Primeiramente separaremos a integral em duas

$$
\frac{d}{d x}\left(\int_{h(x)}^{g(x)} f(t) d t\right)=\frac{d}{d x}\left(\int_{a}^{g(x)} f(t) d t-\int_{a}^{h(x)} f(t) d t\right) \quad \forall a \quad h(x)<a<g(x),
$$

e então calcularemos a derivada

$$
\frac{d}{d x}\left(\int_{h(x)}^{g(x)} f(t) d t\right)=f(g(x)) g^{\prime}(x)-f(h(x)) h^{\prime}(x) .
$$

Assim, usando a equação (2.6.6), as derivadas de $f_{1 \sigma}(\xi)$ e $f_{2 \sigma}(\xi)$ com respeito a $\xi$ são

$$
\begin{gathered}
\frac{d}{d \xi} f_{1 \sigma}(\xi)=\frac{V^{2}}{2 D}\left\{\frac{1}{\xi+\epsilon_{F}-\epsilon_{0 \sigma}-f_{2 \sigma}\left(\xi+\epsilon_{F}\right)}-\frac{1}{\xi-D-\epsilon_{0 \sigma}-f_{2 \sigma}(\xi-D)}\right\} \\
\frac{d}{d \xi} f_{2 \sigma}(\xi)=\frac{V^{2}}{2 D}\left\{\frac{1}{\xi-\epsilon_{F}-\sum_{\sigma} f_{1 \sigma}\left(\xi-\epsilon_{F}\right)}-\frac{1}{\xi-D-\sum_{\sigma} f_{1 \sigma}(\xi-D)}\right\} .
\end{gathered}
$$

Podemos observar que, assumindo $\epsilon_{F}=0$, a solução do problema autoconsistente da equação (2.4.10) corresponde a um pólo da derivada da função $f_{2 \sigma}(\xi)$, equação (2.6.7), no ponto

$$
\xi=\sum_{\sigma} f_{1 \sigma}(\xi)
$$

O pólo da derivada da função $f_{1 \sigma}(\xi)$ está localizado no ponto

$$
\xi-\epsilon_{0 \sigma}=f_{2 \sigma}(\xi)
$$

que neste momento não terá muita importância, entretanto adiante voltaremos a este ponto.

Logo, concluímos que, o valor de energia $\Delta E$ pode ser obtido como o valor da variável $\xi$ da função $f_{1 \sigma}(\xi)$ onde a sua derivada tende a infinito ou no cruzamento de $\xi$ com a função $\sum_{\sigma} f_{1 \sigma}(\xi)$ no espaço $\xi$. 
Um formalismo de operadores de projeção para resolver o Hamiltoniano de Anderson

\section{7}

\section{Função de Green de uma cadeia linear semi-infinita}

A partir deste momento, iniciaremos uma abordagem diferente mostrando que o problema pode ser resolvido calculando formalmente o que seriam as funções de Green de uma rede de Bethe construída a partir dos estados que definem o subespaço $|2\rangle$, obtidos como resultado das sucessivas aplicações do Hamiltoniano $H_{21}$ sobre o estado do subespaço $|1\rangle$, como descrito previamente. Contudo, antes de prosseguirmos estudaremos um problema simples, o qual consiste em uma cadeia linear semi-infinita conforme a figura 2.4, com a finalidade de nos acostumarmos com o formalismo.

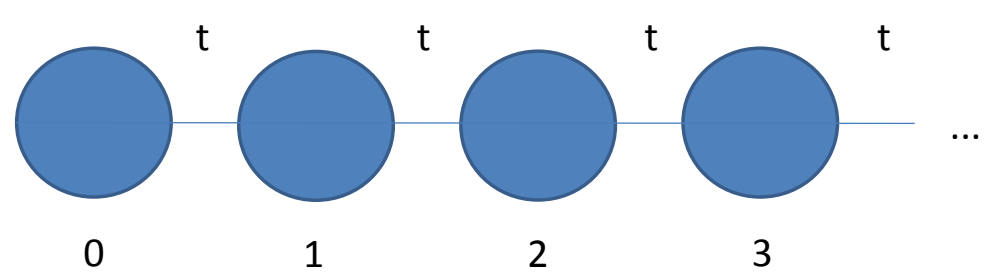

Figura 2.4: Cadeia linear semi-infinita.

A função de Green de uma partícula no sítio $0, G_{00}$, é dada por

$$
G_{00}=g_{0}+g_{0} t G_{10}
$$

onde $t$ é o termo não diagonal entre sítios contíguos, $g_{i}$ é a função de Green de um corpo, do sítio $i$ e $G_{i j}$ a função de Green que descreve a propagação do sítio $i$ ao sítio $j$.

A função de Green no sítio 1,

$$
G_{10}=g_{1} t G_{00}+g_{1} t G_{20},
$$


está conectada ao sítio 0 através do primeiro termo da equação (2.7.2) e ao sítio 2 pelo segundo termo, que representa a ligação entre o sítio 2 e o sítio 0 através do sítio 1.

Continuando para os infinitos elementos da cadeia linear semi-infinita, sempre com o sítio $i$ conectado ao sítio 0 através dos sítios $i-1, i-2, \ldots$, temos

$$
\begin{gathered}
G_{20}=g_{2} t G_{10}+g_{2} t G_{30}, \\
G_{30}=g_{3} t G_{20}+\ldots
\end{gathered}
$$

e assim sucessivamente.

Resolvendo o sistema de equações, (2.7.1), (2.7.2), (2.7.3) e (2.7.4), obtemos a função de Green de uma partícula localizada no sítio 0,

$$
G_{0 \mid 0}=\frac{g_{0}}{1-g_{0} t^{2} \frac{g_{1}}{1-g_{1} t^{2} \frac{g_{2}}{1-\ldots}}},
$$

correspondente a uma fração continuada com uma lei de recorrência dada por

$$
f(n)=\frac{g_{n}}{1-g_{n} t^{2} f(n+1)} .
$$

Novamente no limite em que $N \rightarrow \infty$, temos $f(n)=f(n+1)$ o que nos pemite encontrar autoconsistentemente o valor da função de Green $G_{00}$ de uma semicadeia, dada por

$$
G_{00}=\frac{\omega \pm i \sqrt{4 t^{2}-\omega^{2}}}{2 t^{2}}
$$

onde $g_{i}=\frac{1}{\omega}$. Neste limite, podemos interpretar que ao fazer $f(n)=f(n+1)$, estamos dizendo que deslocar a cadeia por um sítio é equivalente a temos novamente um sítio interagindo com uma cadeia linear semi-infinita, justificando a igualdade.

\section{8}

\section{Termos diagonais}

Utilizando as ideias da seção anterior podemos recuperar o resultado obtido para o cálculo da energia discutido anteriormente. Associamos cada sítio da cadeia linear semi-infinita, figura 2.4 , a cada estado do subespaço $|1\rangle$ e $|2\rangle$, figura 2.3, conforme a figura 2.5. 


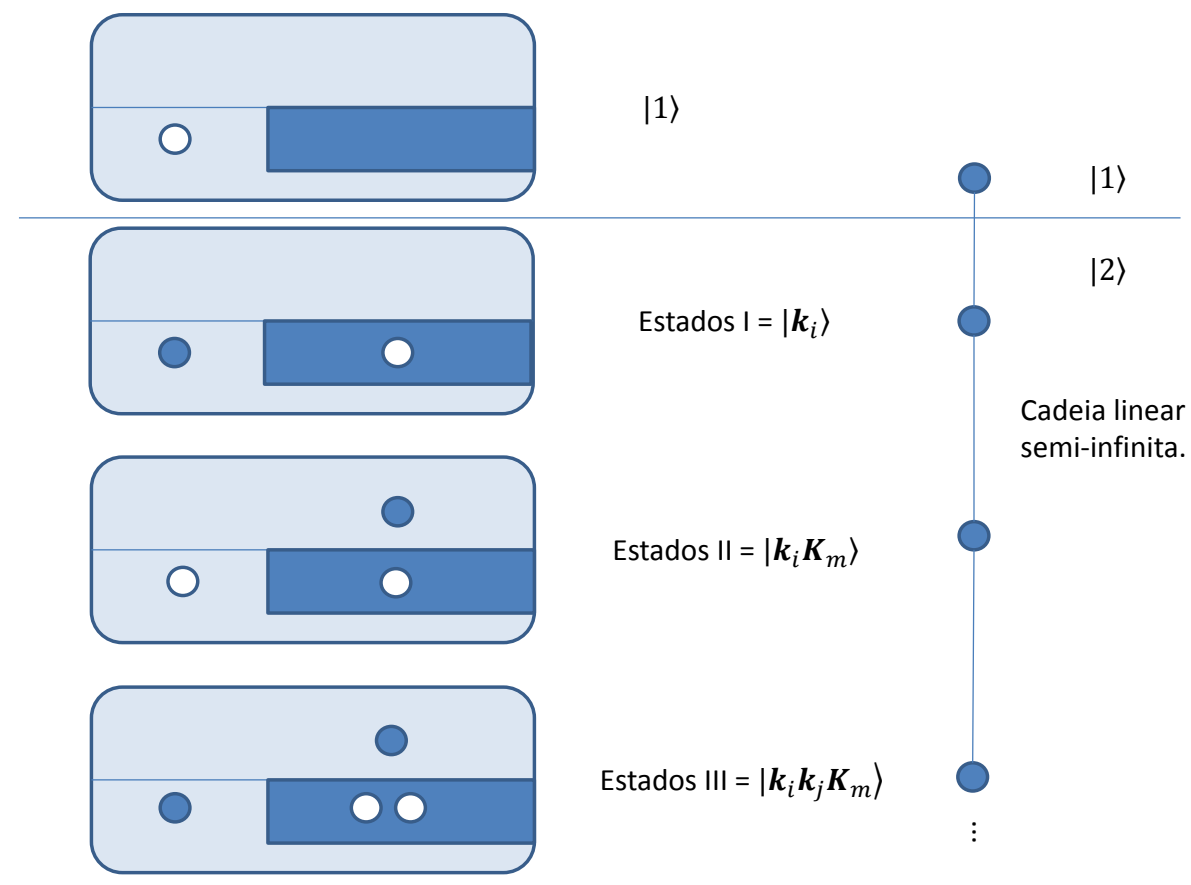

Figura 2.5: Analogia dos subespaços $|1\rangle$ e $|2\rangle$ com a cadeia linear semi-infinita.

Com o formalismo das funções de Green é mais interessante utilizar uma outra notação para os estados do subespaço $|2\rangle$ que anteriormente eram discriminados por estado I, estado II, ... Agora esses estados passam a ser caracterizado pelo vetor número de onda, numa notação mais precisa e concisa. Assim, os estados I nesta nova nomenclatura ficam $\left|\mathbf{k}_{i}\right\rangle$, onde o subíndice $i$ diz respeito a todos os $N$ vetores $\mathbf{k}$ possíveis que descrevem os $N$ elétrons, no caso o momento $\mathbf{k}_{i}$.

Os estados II serão referidos como $\left|\mathbf{k}_{i} \mathbf{K}_{m}\right\rangle$, pois reservamos as letras maiúsculas para os vetores número de onda em que $\epsilon_{\mathbf{K} \sigma}>\epsilon_{F}$. Note que, analogamente ao estado $\left|\mathbf{k}_{i}\right\rangle$, dentre todos os possíveis $\mathbf{K}$, escolhemos o $\mathbf{K}_{m}$. No nome deste estado mantivemos o $\mathbf{k}_{i}$ propositalmente, ou seja, o estado $\left|\mathbf{k}_{i} \mathbf{K}_{m}\right\rangle$, já que esta notação faz referência a todos os números quânticos que caracterizam o estado.

Continuando com o raciocínio, os estado III, que apresentam a retirada de um segundo elétron, são caracterizados nesta nova notação por $\left|\mathbf{k}_{i} \mathbf{k}_{j} \mathbf{K}_{m}\right\rangle$, para $\mathbf{k}_{i} \neq \mathbf{k}_{j}$.

A grande vantagem da implementação desta notação é que ela evidencia certas características que são mais difíceis de visualizar na notação anterior, 
apesar de ser mais simples.

Ao escolhermos um elétron com momento $\mathbf{k}_{i}$, devemos incluir todos os índices $i$ possíveis do conjunto dos $N$ elétrons no mar de Fermi, assim como também para os índices $m$ e $j$. Se em vez de escrevermos os $N$ termos do somatório, escrevermos, por simplicidade, três, podemos representá-los facilmente no diagrama da figura 2.6.

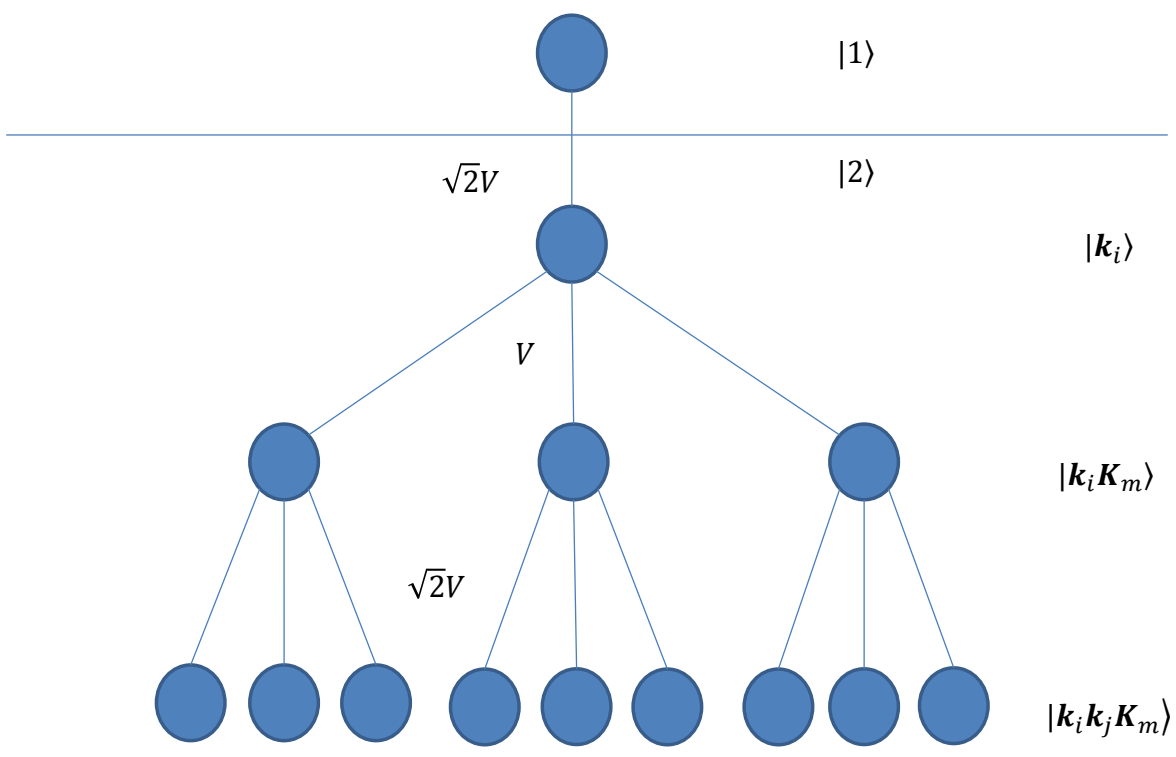

Figura 2.6: Rede de Bethe.

De modo ao estabelecermos o nível hierárquico e junto com o diagrama da figura 2.6, identificamos uma rede de Bethe, ou árvore de Cayley, com número de coordenação igual a quatro no caso da figura 2.6, ou com número de coordenação $N$ para todos os elétrons no mar de Fermi.

Sempre que aniquilamos um elétron no mar de Fermi e criamos um na impureza, aplicando o Hamiltoniano $H_{21}$, formamos um singleto entre o mar de Fermi e o elétron na impureza pois partimos de um singleto, spin total nulo, e o Hamiltoniano de Anderson conserva spin total do sistema. Como consequência devemos multiplicar o termo de hibridização por $\sqrt{2}$, conforme apresentado na figura 2.6, a fim de manter a normalização da função de onda do singleto. Na segunda aplicação do $H_{12}$ isto não é necessário porque a função de partida já está antissimetrizada. Anteriormente, quando calculamos 
a energia através de renormalizações no Hamiltoniano, equação (2.4.8), não precisamos deste artifício pois já levávamos em consideração a contribuição dos diferentes valores de spin.

Agora a comparação com a cadeia linear semi-infinita é imediata. Para aplicar o formalismo das funções de Green discutidas na seção anterior ao nosso problema é necessário extendê-la para uma rede de Bethe.

A função de Green no estado $\left|\mathbf{k}_{i}\right\rangle$ possui a forma

$$
G_{\mathbf{k}_{i} \mid \mathbf{k}_{i}}=g_{\mathbf{k}_{i}}+g_{\mathbf{k}_{i}} \frac{V}{\sqrt{N}} \sum_{\mathbf{K}_{m}} G_{\mathbf{k}_{i} \mathbf{K}_{m} \mid \mathbf{k}_{i}}
$$

onde $g_{\mathbf{k}_{i}}$ é a função de Green de um corpo no estado $\left|\mathbf{k}_{i}\right\rangle$, o termo de hopping é a magnitude do termo de hibridização, $V$, o somatório em $\mathbf{K}_{m}$ é devido a todos os estados acessíveis a partir de $\left|\mathbf{k}_{i}\right\rangle$ e $G_{\mathbf{k}_{i} \mathbf{K}_{m} \mid \mathbf{k}_{i}}$ a função de Green que fornece a propagação de $\left|\mathbf{k}_{i} \mathbf{K}_{m}\right\rangle$ a $\left|\mathbf{k}_{i}\right\rangle$.

Por sua vez,

$$
G_{\mathbf{k}_{i} \mathbf{K}_{m} \mid \mathbf{k}_{i}}=g_{\mathbf{k}_{i} \mathbf{K}_{m}} \frac{\sqrt{2} V}{\sqrt{N}} \sum_{\mathbf{k}_{j}} G_{\mathbf{k}_{i} \mathbf{k}_{j} \mathbf{K}_{m} \mid \mathbf{k}_{i}}+g_{\mathbf{k}_{i} \mathbf{K}_{m}} \frac{V}{\sqrt{N}} G_{\mathbf{k}_{i} \mid \mathbf{k}_{i}}
$$

$\mathrm{e}$

$$
G_{\mathbf{k}_{i} \mathbf{k}_{j} \mathbf{K}_{m} \mid \mathbf{k}_{i}}=g_{\mathbf{k}_{i} \mathbf{k}_{j} \mathbf{K}_{m}} \frac{\sqrt{2} V}{\sqrt{N}} G_{\mathbf{k}_{i} \mathbf{K}_{m} \mid \mathbf{k}_{i}} .
$$

Novamente, $g_{\mathbf{k}_{i} \mathbf{K}_{m}}$ e $g_{\mathbf{k}_{i} \mathbf{k}_{j} \mathbf{K}_{m}}$ são as funções de Green de um corpo nos estados $\left|\mathbf{k}_{i} \mathbf{K}_{m}\right\rangle$ e $\left|\mathbf{k}_{i} \mathbf{k}_{j} \mathbf{K}_{m}\right\rangle$, respectivamente, e $G_{\mathbf{k}_{i} \mathbf{k}_{j} \mathbf{K}_{m} \mid \mathbf{k}_{i}}$ é a função de Green que conecta os estados $\left|\mathbf{k}_{i} \mathbf{k}_{j} \mathbf{K}_{m}\right\rangle$ e $\left|\mathbf{k}_{i}\right\rangle$ através do estado $\left|\mathbf{k}_{i} \mathbf{K}_{m}\right\rangle$. Note que está presente o fator $\sqrt{2}$ discutido há pouco.

Resolvendo o sistema de equações (2.8.1), (2.8.2) e (2.8.3), temos a função de Green no estado imediatamente vizinho ao subespaço $|1\rangle$

$$
G_{\mathbf{k}_{i} \mid \mathbf{k}_{i}}=\frac{g_{\mathbf{k}_{i}}}{1-g_{\mathbf{k}_{i}} \frac{V^{2}}{N} \sum_{\mathbf{K}_{m}} \frac{g_{\mathbf{k}_{i} \mathbf{K}_{m}}}{1-g_{\mathbf{k}_{i} \mathbf{K}_{m}} \frac{2 V^{2}}{N} \sum_{\mathbf{k}_{j}} g_{\mathbf{k}_{i} \mathbf{k}_{j} \mathbf{K}_{m}}}} .
$$

Passando entre os subespaços $|1\rangle$ e $|2\rangle$ devemos somar para todos os valores de $\mathbf{k}_{i}$, multiplicar pelo termo não diagonal que conecta sítios contíguos 
e por $\sqrt{2}$ devido ao singleto, obtendo

$$
\begin{array}{r}
\frac{\sqrt{2} V}{\sqrt{N}} \sum_{\mathbf{k}_{i}} G_{\mathbf{k}_{i} \mid \mathbf{k}_{i}} \frac{\sqrt{2} V}{\sqrt{N}}=\frac{2 V^{2}}{N} \sum_{\mathbf{k}_{i}} G_{\mathbf{k}_{i} \mid \mathbf{k}_{i}}= \\
\frac{2 V^{2}}{N} \sum_{\mathbf{k}_{i}} \frac{g_{\mathbf{k}_{i}}}{1-g_{\mathbf{k}_{i}} \frac{V^{2}}{N} \sum_{\mathbf{K}_{m}} \frac{g_{\mathbf{k}_{i} \mathbf{K}_{m}}}{1-g_{\mathbf{k}_{i} \mathbf{K}_{m}} \frac{2 V^{2}}{N} \sum_{\mathbf{k}_{j}} g_{\mathbf{k}_{i} \mathbf{k}_{j} \mathbf{K}_{m}}}} .
\end{array}
$$

Observe que multiplicamos duas vezes por $\frac{\sqrt{2} V}{\sqrt{N}}$ pois saímos do subespaço $|1\rangle$ percorremos todos os estados do subespaço $|2\rangle$ e voltamos ao subespaço $|1\rangle$.

A função de Green de um corpo em um vetor de estado $|\psi\rangle$ apresenta a forma

$$
g=\left\langle\psi\left|(E-H)^{-1}\right| \psi\right\rangle .
$$

Logo $g_{\mathbf{k}_{i}}, g_{\mathbf{k}_{i} \mathbf{K}_{m}}$ e $g_{\mathbf{k}_{i} \mathbf{k}_{j} \mathbf{K}_{m}}$ podem ser calculados, resultando nas equações (2.8.7), (2.8.8) e (2.8.9).

$$
\begin{gathered}
g_{\mathbf{k}_{i}}=\frac{1}{E-E_{\mathbf{k}_{i}}}=\frac{1}{\Delta E+\epsilon_{\mathbf{k}_{i}}-\epsilon_{0}} \\
g_{\mathbf{k}_{i} \mathbf{K}_{m}}=\frac{1}{E-E_{\mathbf{k}_{i} \mathbf{K}_{m}}}=\frac{1}{\Delta E+\epsilon_{\mathbf{k}_{i}}-\epsilon_{\mathbf{K}_{m}}} \\
g_{\mathbf{k}_{i} \mathbf{k}_{j} \mathbf{K}_{m}}=\frac{1}{E-E_{\mathbf{k}_{i} \mathbf{k}_{j} \mathbf{K}_{m}}}=\frac{1}{\Delta E+\epsilon_{\mathbf{k}_{i}}+\epsilon_{\mathbf{k}_{j}}-\epsilon_{\mathbf{K}_{m}}-\epsilon_{0}}
\end{gathered}
$$

No cálculo da equação (2.8.5) só fomos até o estado três ou $\left|\mathbf{k}_{i} \mathbf{k}_{j} \mathbf{K}_{m}\right\rangle$, contudo notamos claramente a estrutura recorrente de fração continuada, sendo assim, podemos definir uma função de Green de um corpo renormalizada para o estado $\left|\mathbf{k}_{i} \mathbf{k}_{j} \mathbf{K}_{m}\right\rangle, \tilde{g}_{\mathbf{k}_{i} \mathbf{k}_{j} \mathbf{K}_{m}}$, devido a todos os estados que poderiam ser percorridos na rede de Bethe da figura 2.6 como

$$
\tilde{g}_{\mathbf{k}_{i} \mathbf{k}_{j} \mathbf{K}_{m}}=\frac{1}{\Delta E+\epsilon_{\mathbf{k}_{i}}+\epsilon_{\mathbf{k}_{j}}-\epsilon_{\mathbf{K}_{m}}-\epsilon_{0}-f_{2 \sigma}\left(\Delta E+\epsilon_{\mathbf{k}_{i}}+\epsilon_{\mathbf{k}_{j}}-\epsilon_{\mathbf{K}_{m}}-\epsilon_{0}\right)},
$$

com as devidas definições de $f_{1}(\xi)$ e $f_{2 \sigma}(\xi)$ dadas por

$$
\begin{array}{r}
f_{1 \sigma}(\xi)=\frac{2 V^{2}}{N} \sum_{\mathbf{k}} \frac{1}{\xi+\epsilon_{\mathbf{k}}-\epsilon_{0}-f_{2 \sigma}\left(\xi+\epsilon_{\mathbf{k}}\right)} \\
f_{2 \sigma}(\xi)=\frac{V^{2}}{N} \sum_{\mathbf{K}} \frac{1}{\xi-\epsilon_{\mathbf{K}}-f_{1 \sigma}\left(\xi-\epsilon_{\mathbf{K}}\right)} .
\end{array}
$$


A generalização da equação (2.8.5) pode ser reescrita como

$$
\begin{array}{r}
\frac{\sqrt{2} V}{\sqrt{N}} \sum_{\mathbf{k}_{i}} G_{\mathbf{k}_{i} \mid \mathbf{k}_{i}} \frac{\sqrt{2} V}{\sqrt{N}}=\frac{2 V^{2}}{N} \sum_{\mathbf{k}_{i}} G_{\mathbf{k}_{i} \mid \mathbf{k}_{i}}= \\
\frac{2 V^{2}}{N} \sum_{\mathbf{k}_{i}} \frac{g_{\mathbf{k}_{i}}}{1-g_{\mathbf{k}_{i}} \frac{V^{2}}{N} \sum_{\mathbf{K}_{m}} \frac{g_{\mathbf{k}_{i} \mathbf{K}_{m}}}{1-g_{\mathbf{k}_{i} \mathbf{K}_{m}} \frac{2 V^{2}}{N} \sum_{\mathbf{k}_{j}} \tilde{g}_{\mathbf{k}_{i} \mathbf{k}_{j} \mathbf{K}_{m}}}},
\end{array}
$$

note que esta equação é diferente da equação (2.8.5), pois nesta levamos em consideração toda a rede de Bethe através da equação (2.8.10).

Substituindo as equações (2.8.7), (2.8.8) e (2.8.10) na (2.8.12) e omitindo os índices $i, j$ e $m$, temos

$$
\begin{array}{r}
\frac{2 V^{2}}{N} \sum_{\mathbf{k}} \frac{1}{N} \sum_{\mathbf{k}} G_{\mathbf{k} \mid \mathbf{k}}= \\
\Sigma=\frac{2 V^{2}}{N} \sum_{\mathbf{k}} \frac{1}{\Delta E+\epsilon_{\mathbf{k}}-\epsilon_{0}-\frac{V^{2}}{N} \sum_{\mathbf{K}} \frac{1}{\Delta E+\epsilon_{\mathbf{k}}-\epsilon_{\mathbf{K}}-\Sigma}}
\end{array}
$$

que é igual a equação (2.4.8) para o caso sem spin, recuperando o resultado anterior. Isto mostra que a função de Green diagonal sobre o estado $\left|\mathbf{k}_{i}\right\rangle$ calculado na rede de Bethe de coordenação infinita mostrado na figura 2.6 é equivalente ao elemento de matriz do operador da equação (2.2.10), necessário de calcular para encontrar a energia do sistema. Esta proposta de cálculo é muito mais clara que a anterior como ficará em evidência a diante no cálculo de outras contribuições a energia total do estado fundamental.

\section{9}

\section{Termos não diagonais}

Quando calculamos a correção da energia na equação (2.4.8) via sucessivas renormalizações na energia ou através do formalismo das funções de Green na equação (2.8.13), sempre mantivemos os somatórios sobre um mesmo momentum. Isto pode ser visto mais facilmente através da figura 2.6. Nós saímos do subespaço $|1\rangle$ e percorremos todos os estados que constituem o subespaço $|2\rangle$, contudo, não mudamos de ramos na rede de Bethe, ou melhor, escolhido 
um $\mathbf{k}_{i}$ no início do subespaço $|2\rangle$ este mantém-se o mesmo, não vamos a um $\mathbf{k}_{j}$.

Com a finalidade de incorporar esta contribuição, elaboramos o diagrama da figura 2.7. Neste diagrama, no estado em que aniquilarmos o segundo elétron no mar de Fermi, elétron com momentum $\mathbf{k}_{j}$, em vez de retornar pelo caminho que nos levou a este estado, caminho do elétron com momentum $\mathbf{k}_{i}$, voltamos pelo caminho que seria percorrido caso aniquilássemos primeiro o elétron $\mathbf{k}_{j}$, por isso do nome termo não diagonal, pois se escrevêssemos as funções de Green em forma matricial estes termos não estariam na diagonal principal, contrariamente aos termos diagonais obtidos anteriormente.

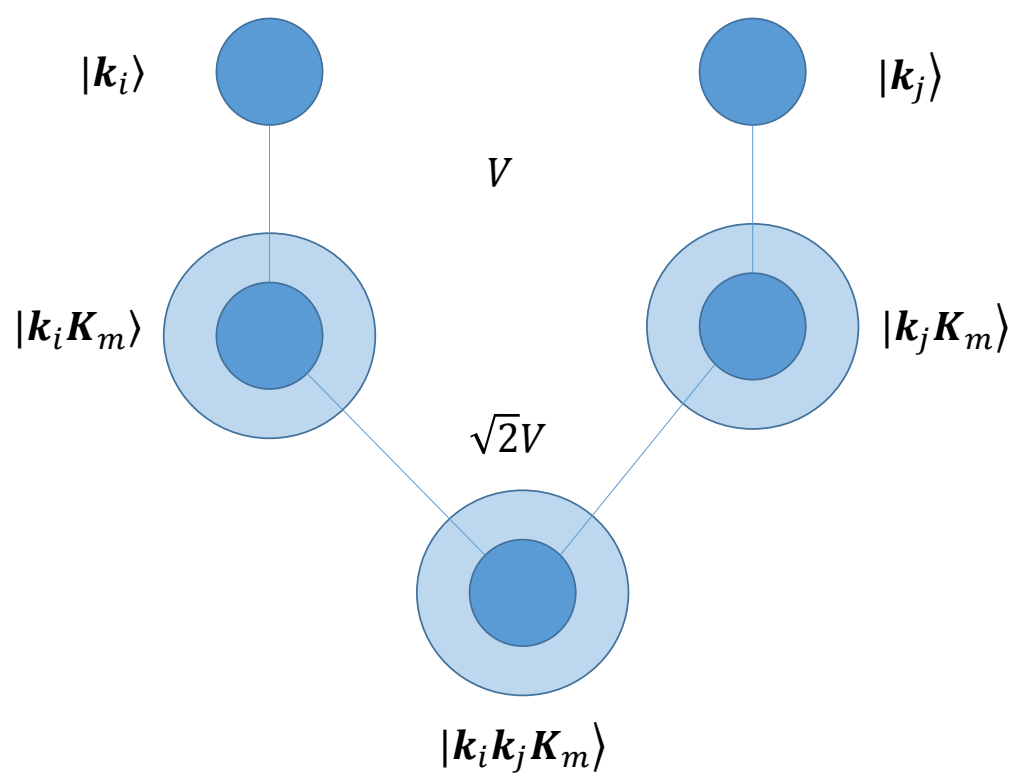

Figura 2.7: Termos não diagonais do subespaço $|2\rangle$. Os estados destacados são renormalizados devido à contribuição dos termos diagonais.

Mas antes de calcularmos estes termos, os estados destacados na figura 2.7, a saber $\left|\mathbf{k}_{i} \mathbf{K}_{m}\right\rangle,\left|\mathbf{k}_{i} \mathbf{k}_{j} \mathbf{K}_{m}\right\rangle$ e $\left|\mathbf{k}_{j} \mathbf{K}_{m}\right\rangle$, devem ser renormalizados por todos os caminhos possíveis oriundos do termo diagonal saindo destes estados. Estas renormalizações ocorrem nas funções de Green de um corpo dos respectivos estados, $\tilde{g}_{\mathbf{k}_{i} \mathbf{K}_{m}}, \tilde{g}_{\mathbf{k}_{i} \mathbf{k}_{j} \mathbf{K}_{m}}$ e $\tilde{g}_{\mathbf{k}_{j} \mathbf{K}_{m}}$, dadas por

$$
\tilde{g}_{\mathbf{k}_{i}}=\frac{1}{E-\tilde{E}_{\mathbf{k}_{i}}}=\frac{1}{\Delta E+\epsilon_{\mathbf{k}_{i}}-\epsilon_{0}-f_{2 \sigma}\left(\Delta E+\epsilon_{\mathbf{k}_{i}}-\epsilon_{0}\right)},
$$


Um formalismo de operadores de projeção para resolver o Hamiltoniano de Anderson

$$
\tilde{g}_{\mathbf{k}_{i} \mathbf{K}_{m}}=\frac{1}{E-\tilde{E}_{\mathbf{k}_{i} \mathbf{K}_{m}}}=\frac{1}{\Delta E+\epsilon_{\mathbf{k}_{i}}-\epsilon_{\mathbf{K}_{m}}-f_{1}\left(\Delta E+\epsilon_{\mathbf{k}_{i}}-\epsilon_{\mathbf{K}_{m}}\right)},
$$

e

$\tilde{g}_{\mathbf{k}_{i} \mathbf{k}_{j} \mathbf{K}_{m}}=\frac{1}{E-\tilde{E}_{\mathbf{k}_{i} \mathbf{k}_{j} \mathbf{K}_{m}}}=\frac{1}{\Delta E+\epsilon_{\mathbf{k}_{i}}+\epsilon_{\mathbf{k}_{j}}-\epsilon_{\mathbf{K}_{m}}-\epsilon_{0}-f_{2 \sigma}\left(\Delta E+\epsilon_{\mathbf{k}_{i}}+\epsilon_{\mathbf{k}_{j}}-\epsilon_{\mathbf{K}_{m}}-\epsilon_{0}\right)}$.

Analogamente ao discutido na secção sobre os termos diagonais, escrevemos as funções de Green como

$$
\begin{gathered}
G_{\mathbf{k}_{i} \mid \mathbf{k}_{j}}=g_{\mathbf{k}_{i}} \frac{V}{\sqrt{N}} \sum_{\mathbf{K}_{m}} G_{\mathbf{k}_{i} \mathbf{K}_{m} \mid \mathbf{k}_{j}} \\
G_{\mathbf{k}_{i} \mathbf{K}_{m} \mid \mathbf{k}_{j}}=\tilde{g}_{\mathbf{k}_{i} \mathbf{K}_{m}} \frac{V}{\sqrt{N}} G_{\mathbf{k}_{i} \mid \mathbf{k}_{j}}+\tilde{g}_{\mathbf{k}_{i} \mathbf{K}_{m}} \frac{\sqrt{2} V}{\sqrt{N}} G_{\mathbf{k}_{i} \mathbf{k}_{j} \mathbf{K}_{m} \mid \mathbf{k}_{j}}, \\
G_{\mathbf{k}_{i} \mathbf{k}_{j} \mathbf{K}_{m} \mid \mathbf{k}_{j}}=\tilde{g}_{\mathbf{k}_{i} \mathbf{k}_{j} \mathbf{K}_{m}} \frac{\sqrt{2} V}{\sqrt{N}} G_{\mathbf{k}_{i} \mathbf{k}_{m} \mid \mathbf{k}_{j}}+\tilde{g}_{\mathbf{k}_{i} \mathbf{k}_{j} \mathbf{K}_{m}} \frac{\sqrt{2} V}{\sqrt{N}} G_{\mathbf{k}_{j} \mathbf{k}_{m} \mid \mathbf{k}_{j}}
\end{gathered}
$$

e

$$
G_{\mathbf{k}_{j} \mathbf{K}_{m} \mid \mathbf{k}_{j}}=\tilde{g}_{\mathbf{k}_{j} \mathbf{K}_{m}} \frac{\sqrt{2} V}{\sqrt{N}} G_{\mathbf{k}_{i} \mathbf{k}_{j} \mathbf{K}_{m} \mid \mathbf{k}_{j}}+\tilde{g}_{\mathbf{k}_{j} \mathbf{K}_{m}} \frac{V}{\sqrt{N}} G_{\mathbf{k}_{j} \mid \mathbf{k}_{j}}
$$

Resolvendo o sistema de equações (2.9.4), (2.9.5), (2.9.6) e (2.9.7), obtemos:

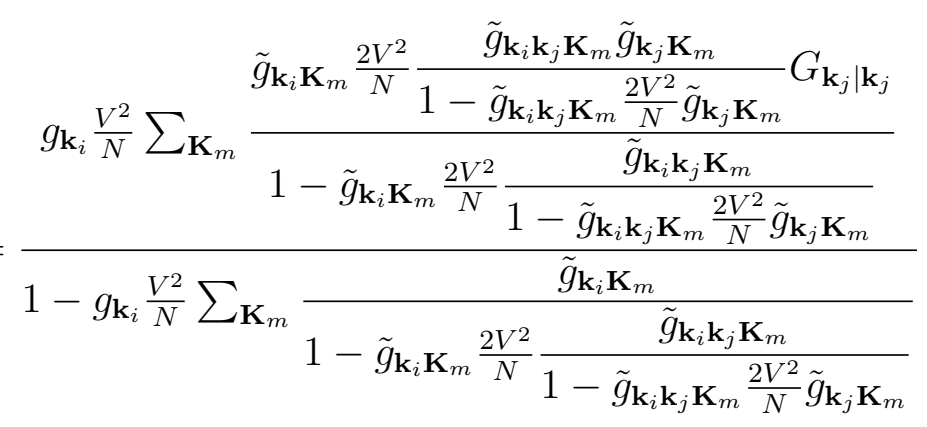

Como os somatórios são realizados sobre $N$ termos, somente os temos que envolvem somatórios sobrevivem na equação (2.9.8), resultando em

$$
G_{\mathbf{k}_{i} \mid \mathbf{k}_{j}}=\frac{\frac{2 V^{4}}{N^{2}} g_{\mathbf{k}_{i}} \sum_{\mathbf{K}_{m}} \tilde{g}_{\mathbf{k}_{i} \mathbf{K}_{m}} \tilde{g}_{\mathbf{k}_{i} \mathbf{k}_{j} \mathbf{K}_{m}} \tilde{g}_{\mathbf{k}_{j} \mathbf{K}_{m}} G_{\mathbf{k}_{j} \mid \mathbf{k}_{j}}}{1-g_{\mathbf{k}_{i}} \frac{V^{2}}{N} \sum_{\mathbf{K}_{m}} \tilde{g}_{\mathbf{k}_{i} \mathbf{K}_{m}}}
$$

Somando para todo $\mathbf{k}_{i}$ e $\mathbf{k}_{j}$, multiplicando por $\frac{2 V^{2}}{N}$, substituindo as equações (2.9.1), (2.9.2) e (2.9.3) na equação (2.9.9) e omitindo os índices 
$i, j$ e $m$ obtemos a contribuiçã do termo não diagonal

$$
\begin{array}{r}
\frac{2 V^{2}}{N} \sum_{\mathbf{k}} \sum_{\mathbf{k}^{\prime}} G_{\mathbf{k} \mid \mathbf{k}},= \\
\frac{4 V^{6}}{N^{3}} \sum_{\mathbf{k}} \sum_{\mathbf{k}^{\prime}} \sum_{\mathbf{K}} \frac{1}{\Delta E+\epsilon_{\mathbf{k}}-\epsilon_{\mathbf{K}}-f_{1}\left(\Delta E+\epsilon_{\mathbf{k}}-\epsilon_{\mathbf{K}}\right)} \\
\frac{1}{\Delta E+\epsilon_{\mathbf{k}}+\epsilon_{\mathbf{k}},-\epsilon_{\mathbf{K}}-\epsilon_{0}-f_{2 \sigma}\left(\Delta E+\epsilon_{\mathbf{k}}+\epsilon_{\mathbf{k}},-\epsilon_{\mathbf{K}}-\epsilon_{0}\right)} \\
\frac{1}{\Delta E+\epsilon_{\mathbf{k}},-\epsilon_{\mathbf{K}}-f_{1}\left(\Delta E+\epsilon_{\mathbf{k}},-\epsilon_{\mathbf{K}}\right)} \\
\frac{1}{\Delta E+\epsilon_{\mathbf{k}}-\epsilon_{0}-f_{2 \sigma}\left(\Delta E+\epsilon_{\mathbf{k}}-\epsilon_{0}\right)} \frac{1}{\Delta E+\epsilon_{\mathbf{k}},-\epsilon_{0}-f_{2 \sigma}\left(\Delta E+\epsilon_{\mathbf{k}},-\epsilon_{0}\right)} .
\end{array}
$$

Passando ao contínuo, para uma banda de condução plana, como no caso do termo diagonal, a equação (2.9.10) fica

$$
\begin{array}{r}
\frac{4 V^{6}}{(2 D)^{3}} \int_{-D}^{\epsilon_{F}} d x \int_{-D}^{\epsilon_{F}} d y \int_{\epsilon_{F}}^{D} d z \\
\frac{1}{\xi+x-z-f_{1}(\xi+x-z)} \frac{1}{\xi+y-z-f_{1}(\xi+y-z)} \\
\frac{1}{\xi+x+y-z-\epsilon_{0}-f_{2 \sigma}\left(\xi+x+y-z-\epsilon_{0}\right)} \\
\frac{1}{\xi+x-\epsilon_{0}-f_{2 \sigma}\left(\xi+x-\epsilon_{0}\right)} \frac{1}{\xi+y-\epsilon_{0}-f_{2 \sigma}\left(\xi+y-\epsilon_{0}\right)},
\end{array}
$$

onde vemos que os somatórios da equação (2.9.10) tornam-se uma integral tripla.

Mostraremos no capítulo seguinte que para os parâmetros usuais esta contribuição é desprezível quando comparada com o termo diagonal, já que $V \ll D$.

\subsection{0}

\section{Resumo do capítulo}

Neste capítulo apresentamos o método proposto para a a obtenção da energia do estado fundamental do Hamiltoniano da impureza magnética de Anderson, equação (2.3.1). O método consiste em projetar o espaço de Hilbert em dois subespaços convenientes de forma que possamos obter a solução 
de uma forma mais simples. Ao projetar o Hamiltoniano no subespaço $|1\rangle$ obtemos uma nova equação de Schrödinger, equação (2.2.9), com os mesmos autovalores do Hamiltoniano original.

O valor da correção à energia produzida pela interação entre a impureza e o mar de Fermi foi obtida utilizando sucessivas renormalizações da mesma. Para uma banda de condução plana, definimos duas funções $f_{1 \sigma}(\xi)$ e $f_{2 \sigma}(\xi)$, equações (2.5.2), com as quais obtemos a energia através de um cálculo autoconsisteste e analisamos o comportamento de suas derivadas, equações (2.6.7), e as divergências delas, equações (2.6.9) e (2.6.8).

Através do formalismo das funções de Green, obtemos o mesmo resultado para a correção da energia, mostrando a equivalência do problema ao de uma rede de Bethe com número de coordenação infinito. Desta forma é possível o cálculo dos termos diagonais, equação (2.8.13), os termos não diagonais, equação (2.9.10), e correções de ordem superior em potências de $V$. 


\section{3}

\section{Solução Numérica}

\section{1}

\section{Algoritmo}

Para resolver numericamente as integrais da equação (2.6.2) distribuiremos os pontos em uma região entre $-2 D<x<2 D$; note que a banda plana estende-se de $-D<x<D$. A distribuição mais simples entre os pontos seria uma distribuição equidistante, contudo, para que possamos ter uma boa resolução na região de interesse utilizaremos uma distribuição dada por

$$
x=\sum_{i}^{N_{p}} 2 D\left(\frac{1-r^{i}}{1-r^{k}}\right),
$$

onde $r$ é um parâmetro que controla a densidade de pontos na região de interesse, $k$ é a metade do número de pontos $N_{p}$ que desejamos distribuir e $x$ é a localização de cada ponto.

A região de interesse em que desejamos aumentar a resolução, ou melhor, aumentar o número de pontos localizados, é aquela em que ocorre a intersecção de $\sum_{\sigma} f_{1 \sigma}(\xi)$ com $f(\xi)=\xi$, para tal utilizamos o valor de $r$ de 1.01. Contudo, ao aumentarmos $r$, nós aumentamos a densidade dos pontos em torno do ponto $x=0$, por isso deve ser somado uma constante à equação (3.1.1) para que a região de interesse coincida com a região de maior densidade dos pontos, constante essa que deve ser ajustada manualmente para cada cálculo.

Com os pontos distribuídos calculamos $f_{1 \sigma}(\xi)$ e $f_{2 \sigma}(\xi)$ em cada um dos pontos distribuídos, $x_{i}$. Inicialmente para calcular $f_{1 \sigma}(\xi)$ tomamos o valor de $f_{2 \sigma}(\xi)$ igual a zero e calculamos a primeira iteração do cálculo, analogamente para $f_{2 \sigma}(\xi)$; note que esta primeira iteração pode ser resolvida analiticamente. Depois, na próxima iteração, utilizamos os resultados já obtidos na iteração anterior e assim sucessivamente até a convergência do cálculo. 
Para assegurar convergência, o resultado de cada iteração é mesclado ao resultado da iteração anterior. Esta técnica evita que o resultado de nosso cálculo autoconsistente fuja da região de convergência da função, da bacia de atração da solução.

Como as funções $f_{1 \sigma}(\xi)$ e $f_{2 \sigma}(\xi)$ apresentam inúmeros pólos, nós deslocamos as funções no plano complexo adicionando i $\eta$ ao denominador, onde $i=\sqrt{-1}$. Por simplicidade, escolhemos ilustrar o caso sem o campo magnético, equação (2.6.2),

$$
\begin{aligned}
f_{1 \sigma}(\xi) & =\frac{V^{2}}{2 D} \int_{-D}^{\epsilon_{F}} \frac{1}{\xi+x-\epsilon_{0 \sigma}-f_{2 \sigma}(\xi+x)+i \eta} d x \\
f_{2 \sigma}(\xi) & =\frac{V^{2}}{2 D} \int_{\epsilon_{F}}^{D} \frac{1}{\xi-x-\sum_{\sigma} f_{1 \sigma}(\xi-x)+i \eta} d x
\end{aligned}
$$

Assim, saímos do eixo real onde os pólos estão localizados e resolvemos a integração no plano complexo. Se tomarmos a parte imaginária $\eta$ suficientemente pequena no limite em que $\eta \rightarrow 0$ as equações (3.1.2) aproximam-se das equações originais que queremos conhecer, (2.6.2).

No cálculo numérico escolhemos $\eta$ de forma que possamos ajustar cada pico ou variação das funções $f_{1 \sigma}(\xi)$ e $f_{2 \sigma}(\xi)$ com pelo menos dez pontos, de modo que tenhamos uma boa resolução para discenir um comportamento real da função de possíveis erros numéricos.

\section{2}

\section{Resultados}

Os resultados serão expressos em termos da função de hibridização $\Delta$, onde

$$
\Delta=\frac{\pi V^{2}}{2 D} .
$$

Isto é conveniente pois sabemos que a temperatura Kondo apresenta uma dependência da função de hibridização dada por

$$
T_{K} \cong D e^{-\frac{\pi \epsilon_{0}}{2 \Delta}}
$$

como pode ser obtido a partir do poor Man's scaling do modelo de Anderson, (4) e (20). 
Mostar que nossos resultados estão de acordo com equação (3.2.2) é o grande objetivo do presente trabalho, uma vez que a equação (3.2.2) corresponde à expressão para a temperatura Kondo, obtida por várias outras propostas teóricas hoje bem estabelecidas, no limite de $U$ tendendo a infinito. A obtenção deste resultado permitirá analisar a correção de nossa teoria.

A função $\sum_{\sigma} f_{1 \sigma}(\xi)$ está apresentada na figura 3.1. Nesta figura a intersecção entre as funções $\sum_{\sigma} f_{1 \sigma}(\xi)$ e $f(\xi)=\xi$ satisfaz a condição da equação (2.4.10) nos fornecendo o resultado autoconsistente da correção da energia $\Delta E$.

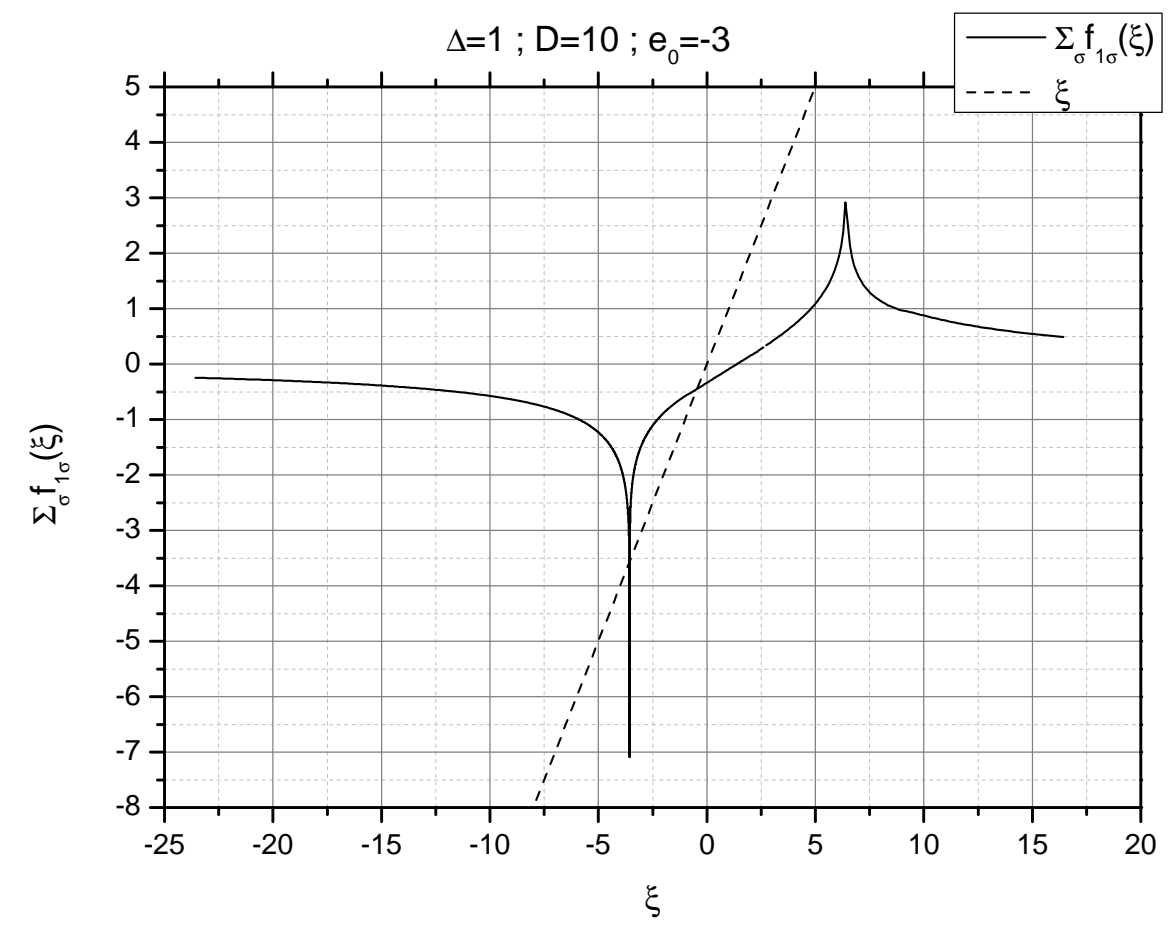

Figura 3.1: A linha contínua apresenta a parte real da função $\sum_{\sigma} f_{1 \sigma}(\xi)$ e a linha tracejada a $f(\xi)=\xi$. A intersecção entre as mesmas fornece a correção da energia $\Delta E$. Como buscamos a energia do estado fundamental, a intersecção que fornece a correção da energia é aquela de menor valor. $\mathrm{O}$ valor de $\eta$ utilizado é de $\eta=10^{-7}$.

Ampliando o gráfico de $\sum_{\sigma} f_{1 \sigma}(\xi)$, figura 3.2 , vemos a presença de uma microestrutura que é responsável pela determinação do valor correto da correção da energia, ligeiramente diferente do valor que poderia ter sido encontrado ingenuamente a partir da figura 3.1. 


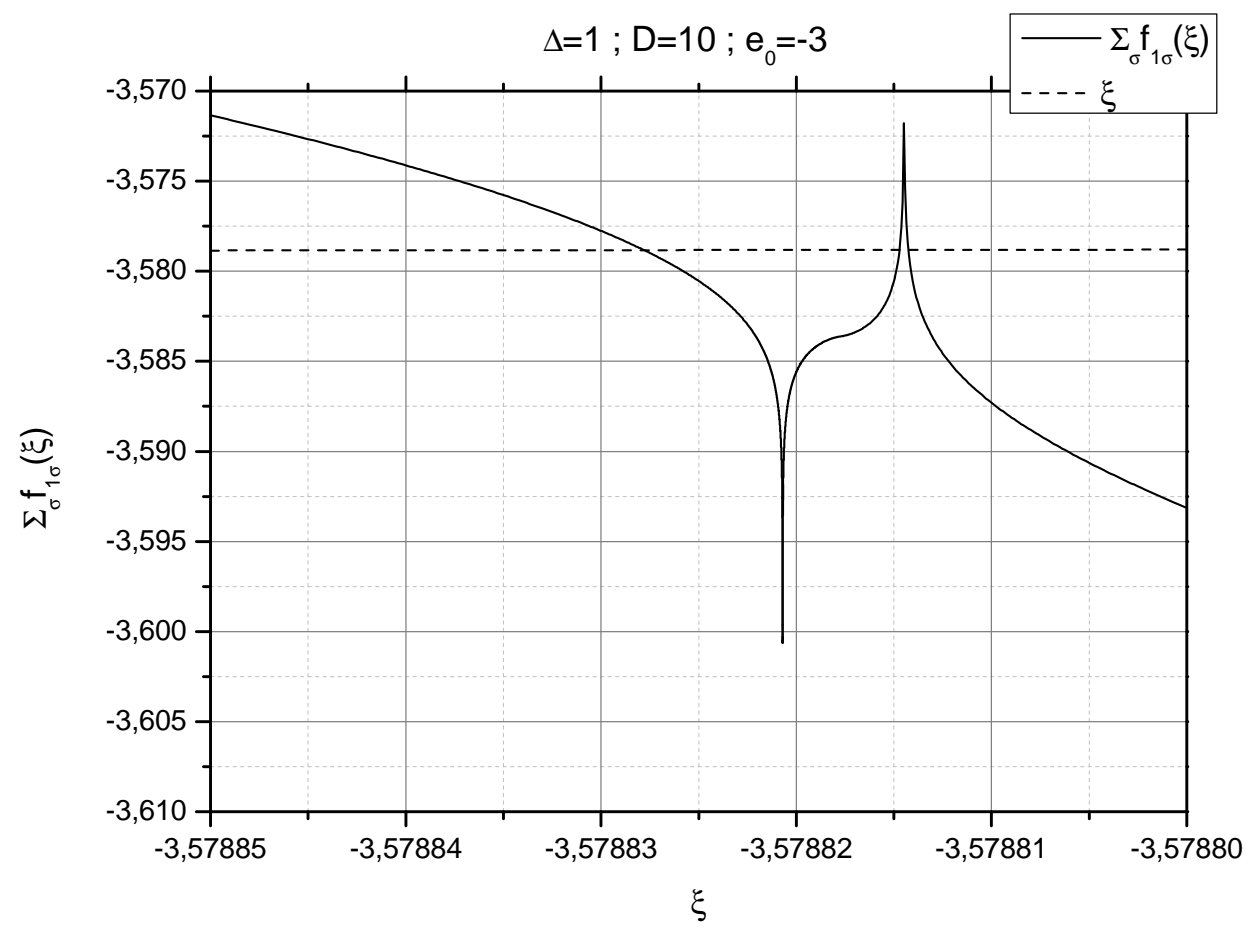

Figura 3.2: Ampliação do gráfico da figura 3.1 na região de corte entre as funções parte real $\sum_{\sigma} f_{1 \sigma}(\xi)$ e $f(\xi)=\xi$.

Por sua vez, a função $f_{2 \sigma}(\xi)$ encontra-se na figura 3.3, onde também é apresentado a função $f(\xi)=\xi-\epsilon_{0}$, cuja intersecção com a função $f_{2 \sigma}(\xi)$ apresenta uma divergência da derivada da função $\sum_{\sigma} f_{1 \sigma}(\xi)$ com respeito a $\xi$, como discutido no capítulo anterior, equação (2.6.9).

Numericamente, vemos que o valor de $\xi$ em que é satisfeita a relação $\sum_{\sigma} f_{1 \sigma}(\xi)=\xi$, correspondente ao valor da correção da energia $\Delta E$ que queremos, é o mesmo em que ocorre a intersecção de $f_{2 \sigma}(\xi) \operatorname{com} \xi-\epsilon_{0}$, de forma que a solução para o problema pode ser obtida pela divergência na derivada de $f_{1 \sigma}(\xi)$, na derivada de $f_{2 \sigma}(\xi)$, na intersecção de $\sum_{\sigma} f_{1 \sigma}(\xi)=\xi$ ou na intersecção de $f_{2 \sigma}(\xi)=\xi-\epsilon_{0}$, pois correspondem ao mesmo ponto, figura 3.4 .

Mostramos que a condição $\sum_{\sigma} f_{1 \sigma}(\xi)=\xi$ surge de ter assumido o mar de Fermi no seu estado fundamental e a impureza vazia como base para definir o subespaço $|1\rangle$. Entretanto, caso tivéssemos escolhido como subespaço $|1\rangle$ o estado com a impureza com um elétron e o mar de Fermi, a condição que nos forneceria a correção da energia seria justamente $f_{2 \sigma}(\xi)=\xi-\epsilon_{0}$. Ponto 


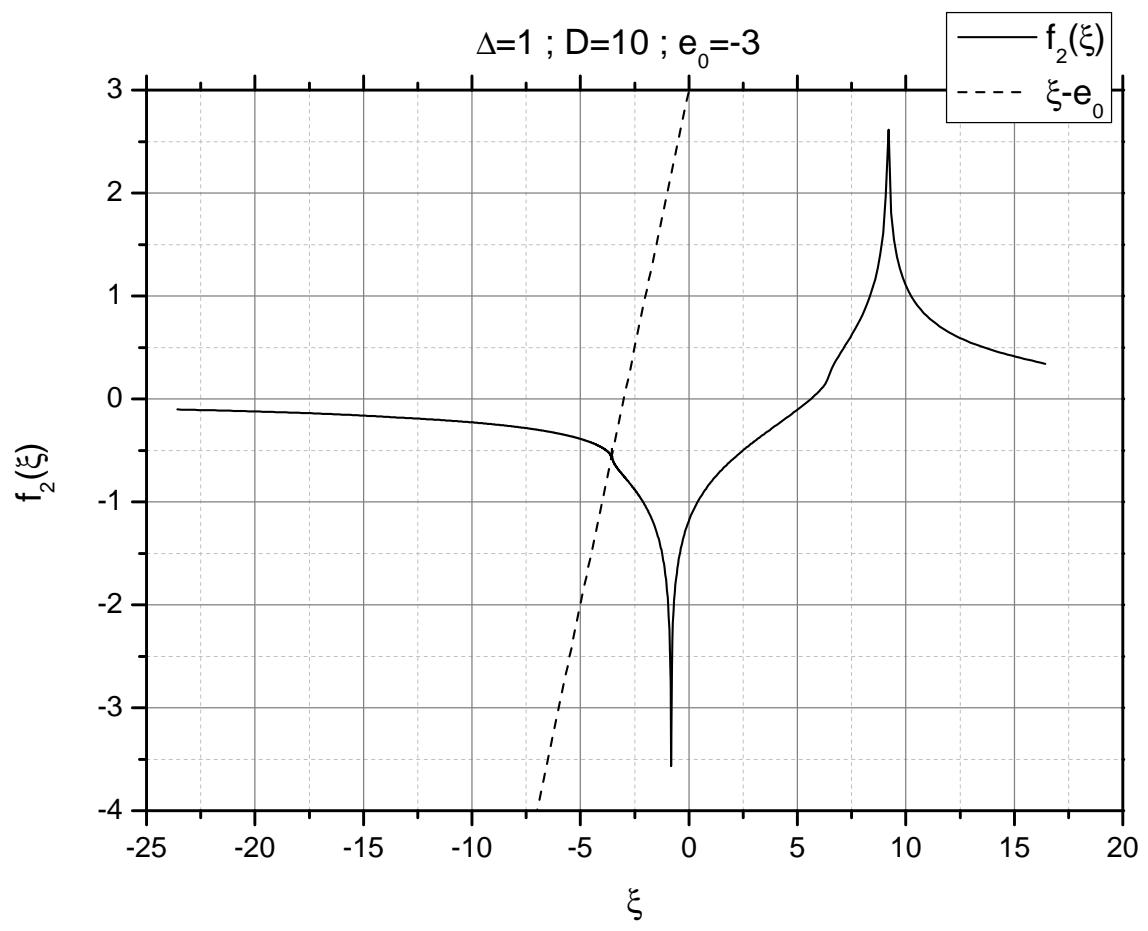

Figura 3.3: A linha contínua apresenta parte real da função $f_{2 \sigma}(\xi)$ e a linha tracejada a função $f(\xi)=\xi-\epsilon_{0}$.

onde a derivada da função $\sum_{\sigma} f_{1 \sigma}(\xi)$ diverge e não onde a derivada da função $f_{2 \sigma}(\xi)$ diverge. Como a correção da energia não pode depender da autofunção que escolhemos para o subespaço $|1\rangle$, obter a correção da energia através da divergência da derivada de $\sum_{\sigma} f_{1 \sigma}(\xi)$ ou $f_{2 \sigma}(\xi)$ é equivalente.

Como conhecemos as funções $\sum_{\sigma} f_{1 \sigma}(\xi)$ e $f_{2 \sigma}(\xi)$, podemos calcular a contribuição do termo não diagonal realizando a integral tripla da equação (2.9.11) numericamente. A figura 3.5 apresenta o gráfico do termo não diagonal.

Analisando a figura 3.5, vemos que podemos desprezar o termo não diagonal visto que sua contribuição ao cálculo da correção da energia frente ao termo diagonal é despezível. Isto consiste em considerar que o Hamiltoniano da equação (2.2.10) pode ser expresso em uma representação em que é diagonal.

O estado de carga na impureza pode ser obtido via equação (1.4.5), derivando $\Delta E$ com respeito a $\epsilon_{0}$. Calculando o valor $\Delta E$ em função da energia local da impureza $\epsilon_{0}$, obtemos o gráfico da figura 3.6. 


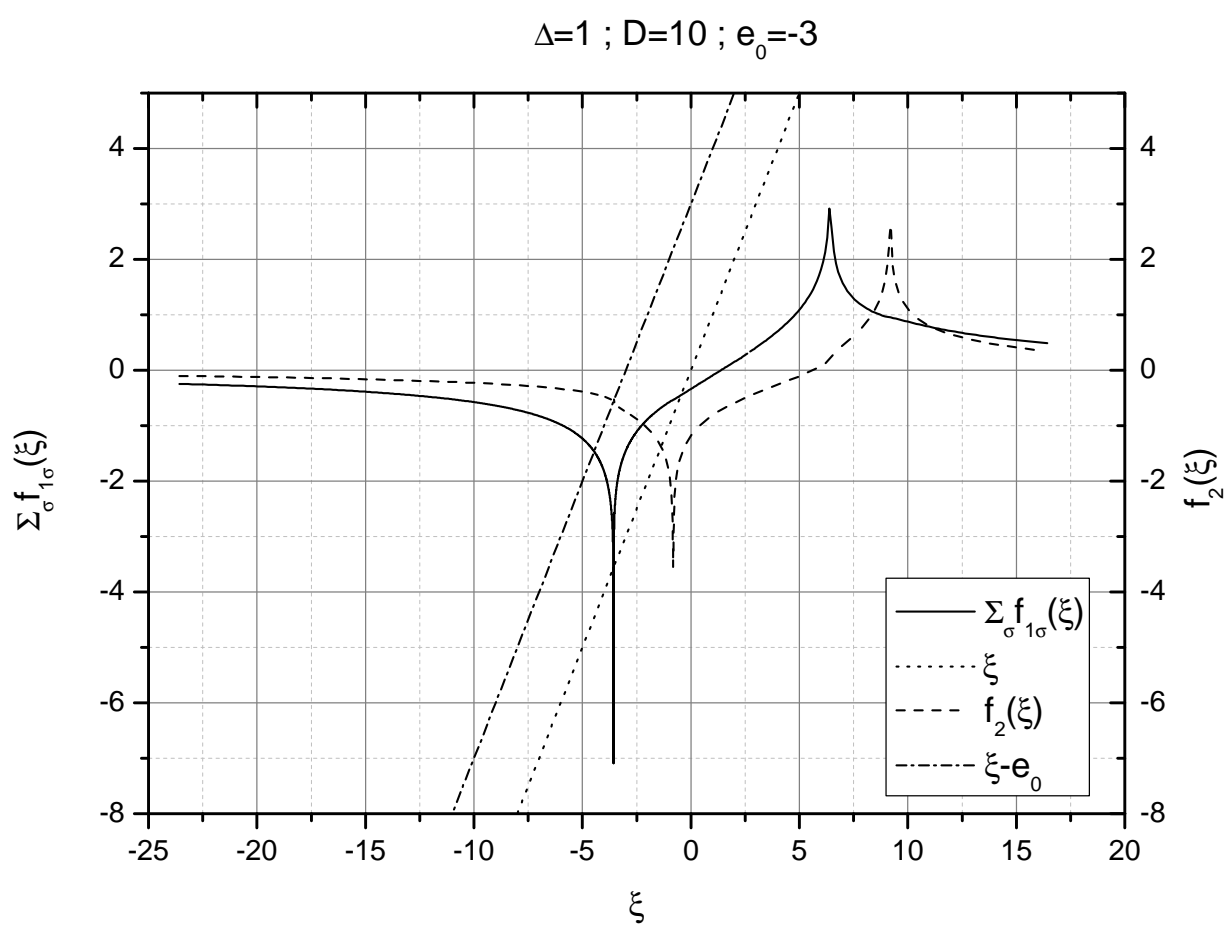

Figura 3.4: Gráfico que apresenta a intersecção da parte real $\sum_{\sigma} f_{1 \sigma}(\xi) \mathrm{com}$ $f(\xi)=\xi$ e a intersecção da parte real $f_{2 \sigma}(\xi)$ e $f(\xi)=\xi-\epsilon_{0}$. A intersecção ocorre no mesmo ponto, $\xi=\Delta E$, correspondente a correção da energia.

Quando o estado localizado está bem por baixo do nível de Fermi a energia tem um comportamento linear e para valores maiores, na região de flutuação de carga, a derivada diminui e eventualmente torna-se zero quando $\epsilon_{0}$ está bem por cima do nível de Fermi.

Calculando a derivada $\frac{\partial}{\partial \epsilon_{0}} \Delta E$ numericamente, obtemos a carga como função de $\epsilon_{0}$ como mostrado no gráfico da figura 3.7, refletindo que na região do bloqueamento de Coulomb, $\epsilon_{0} \ll \epsilon_{F}$, o número de ocupação da impureza é $\left\langle n_{0}\right\rangle=1$, contudo, quando $\epsilon_{0} \lesssim \epsilon_{F}$, na região de flutuação de carga, $\left\langle n_{0}\right\rangle<1$, tendendo para zero quando $\epsilon_{0}>\epsilon_{F}$.

Calculamos a correção da energia para diferentes valores de campo magnético como apresentado na figura 3.8. Nela realizamos um ajuste polinomial e com o coeficiente quadrático podemos obter a susceptibilidade magnética e consequentemente a temperatura Kondo, como já exposto no primeiro capítulo, equações (1.4.8) e (1.4.10). 
Um formalismo de operadores de projeção para resolver o Hamiltoniano de Anderson

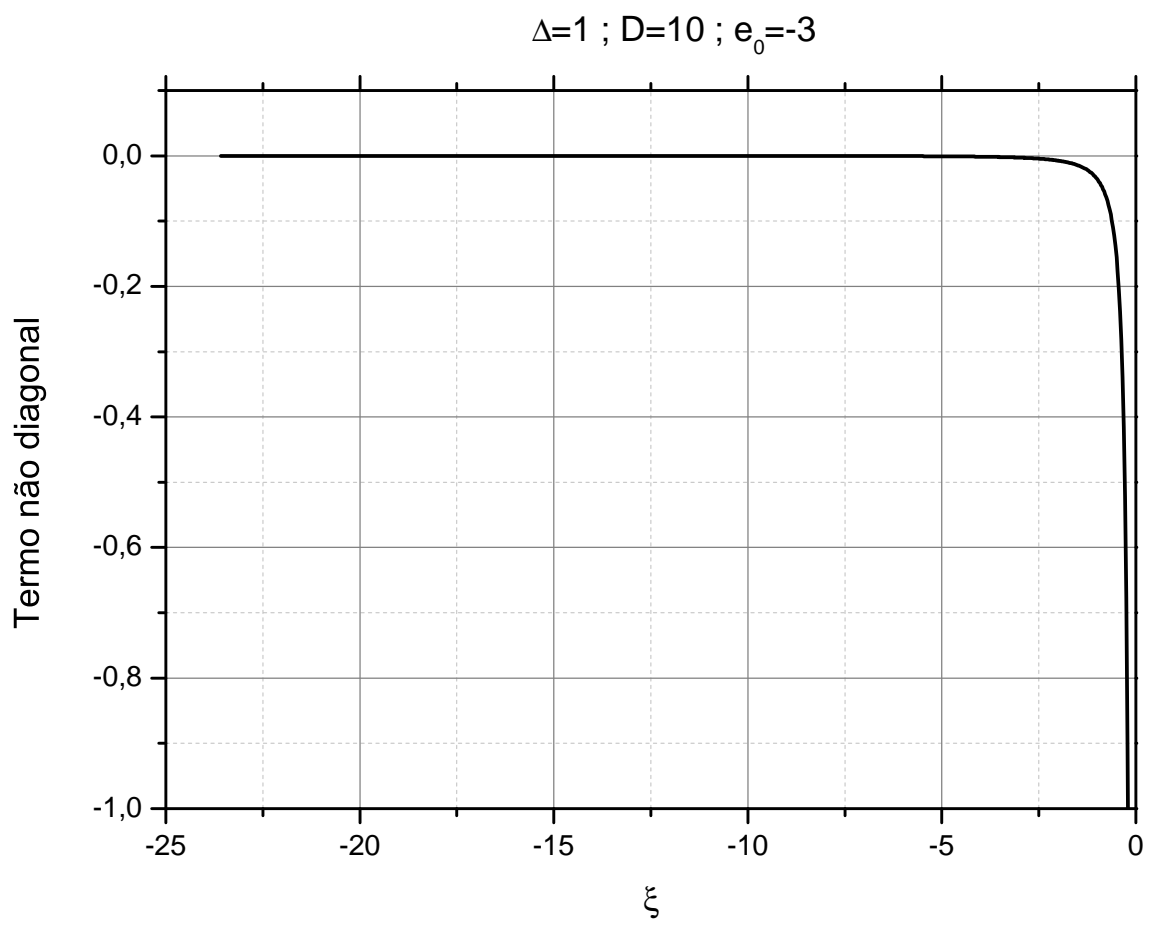

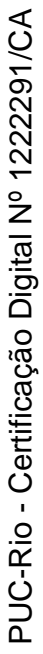

Figura 3.5: Termo não diagonal.

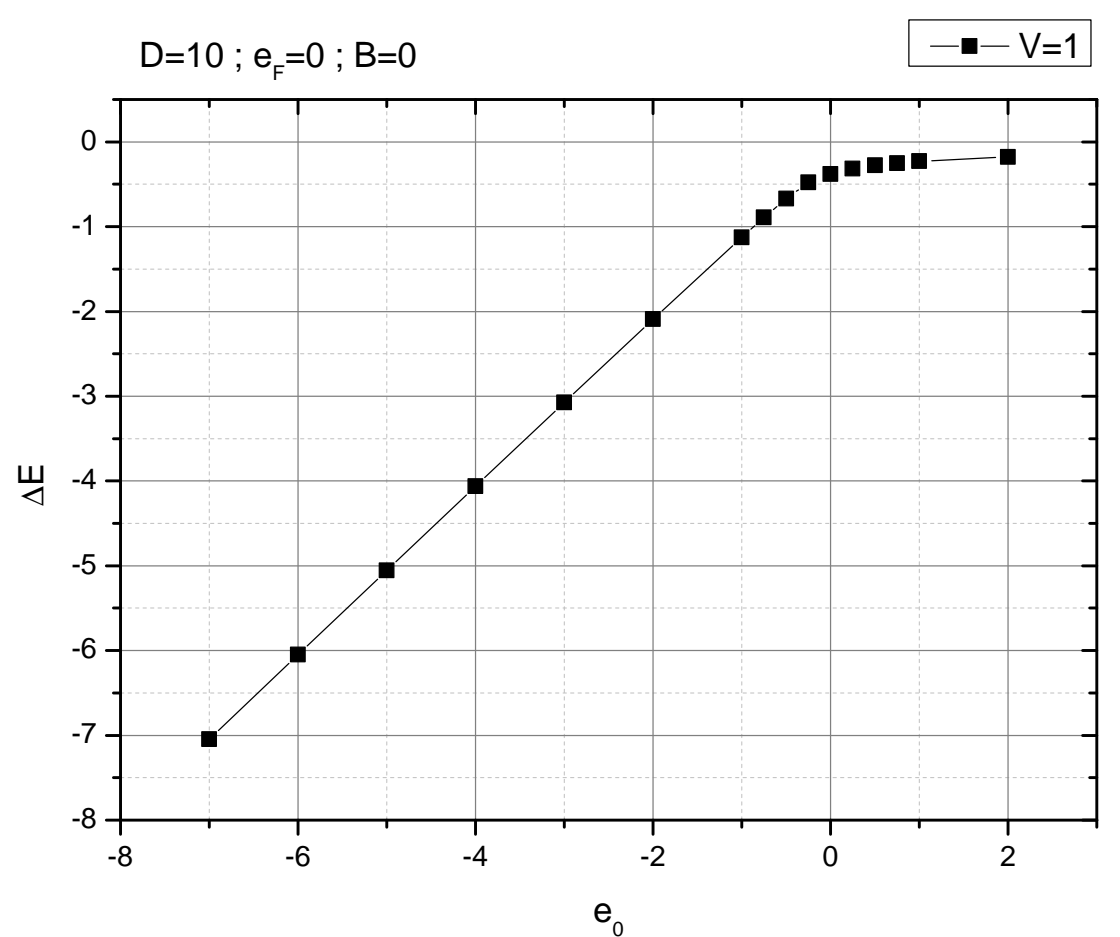

Figura 3.6: Correção da energia como função da energia da impureza. 


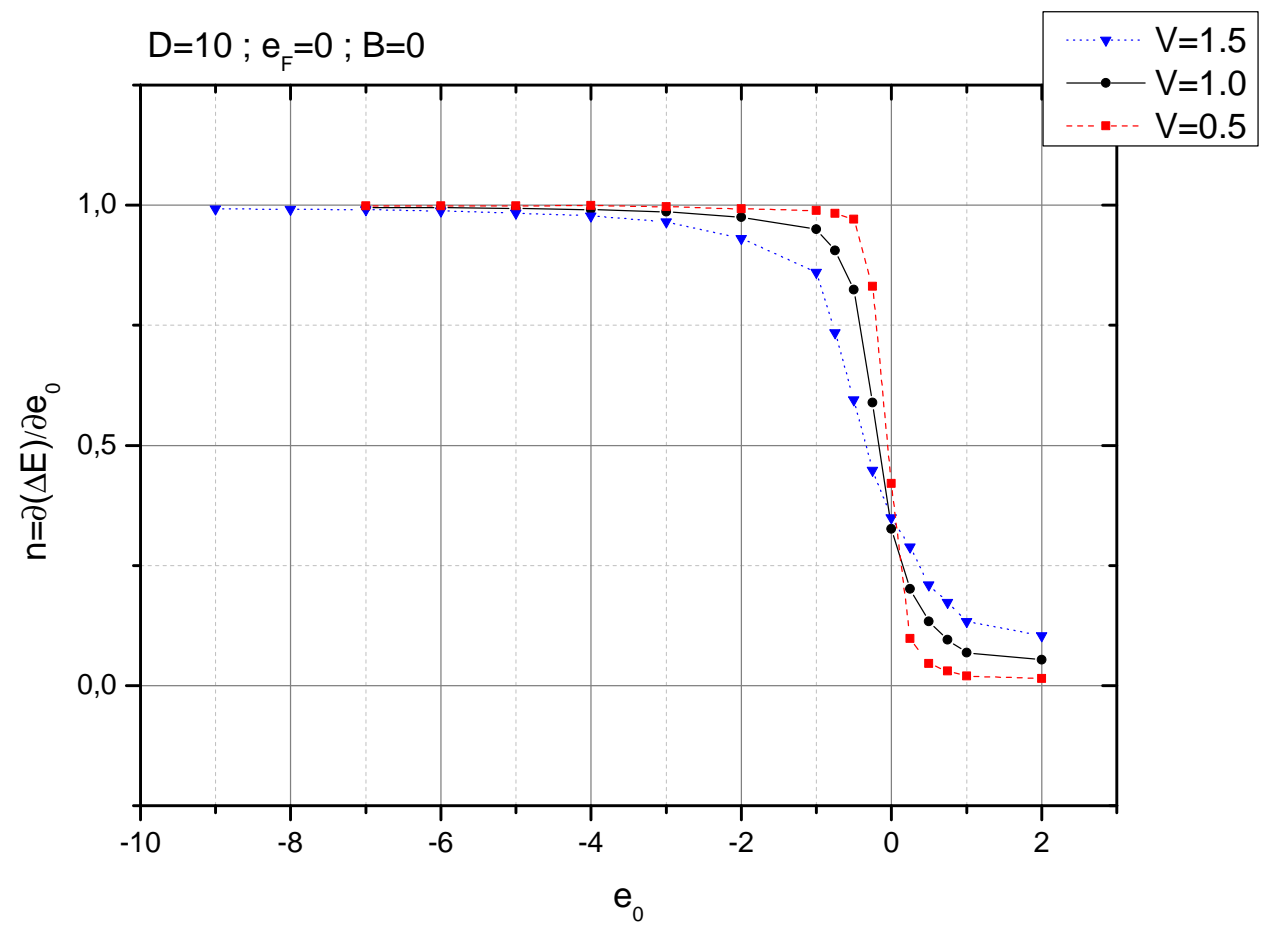

Figura 3.7: Número de ocupação eletrônica na impureza como função da energia da impureza.

Se derivamos numericamente os dados da figura 3.8, equação (1.4.7), obtemos a magnetização na impureza

$$
M=-\frac{\partial}{\partial B} \Delta E,
$$

conforme mostramos na figura 3.9.

A partir do gráfico da magnetização como função do campo magnético externo, exposto na figura 3.9, podemos observar que a magnetização é uma função anti-simétrica e linear com o campo magnético para pequenos valores do campo, como explicitado na figura 3.10, onde realizamos um ajuste linear.

A susceptibilidade magnética da impureza

$$
\chi=\frac{\partial^{2}}{\partial B^{2}} \Delta E
$$

é calculada através do coeficiente quadrático de um ajuste polinomial dos dados da figura 3.8, no limite em que o campo magnético tende a zero temos o valor da susceptibilidade magnética da impureza a campo zero, necessária para o cálculo da temperatura Kondo. 
Um formalismo de operadores de projeção para resolver o Hamiltoniano de Anderson

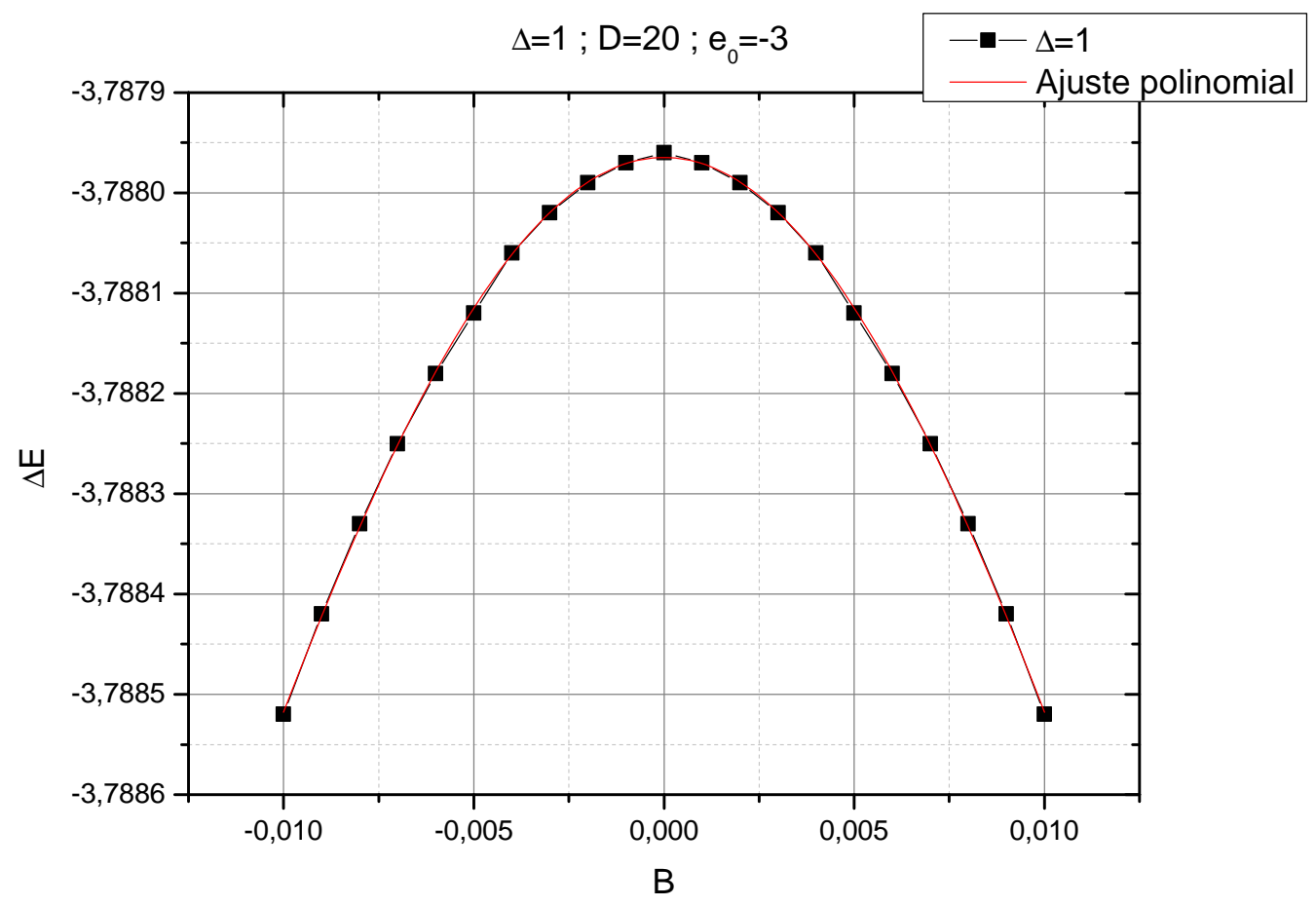

Figura 3.8: Cálculo da correção da energia para diferentes valores do campo magnético externo e o ajuste polinomial.

Por fim, analisamos a dependência da temperatura Kondo conforme a razão da energia da impureza magnética dividida pela função de hibridização, $\frac{\epsilon_{0}}{\Delta}$. A figura 3.11 apresenta o logarítimo de $\frac{T_{K}}{D}$ como função de $\frac{\epsilon_{0}}{\Delta}$, onde comparamos o valor de $\frac{\pi}{2} \approx 1,57079 \ldots$ com o resultado do coeficiente angular do ajuste linear na figura 3.11 de $1.518 \pm 0,065$, de forma que nossos resultados estão de acordo com a equação (3.2.2) conforme exposto na figura 3.11. 
Um formalismo de operadores de projeção para resolver o Hamiltoniano de Anderson

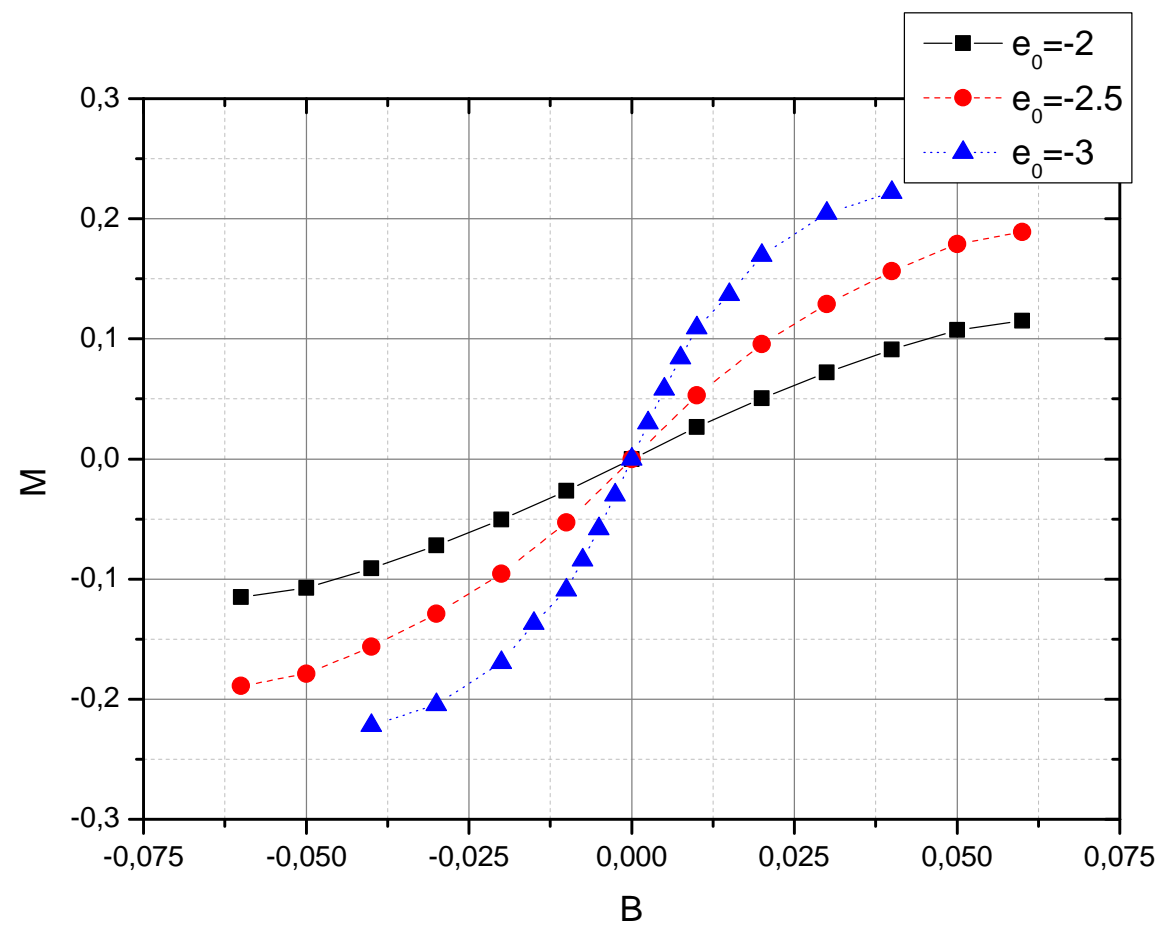

Figura 3.9: Magnetização da impureza como função do campo magnético externo. Obtida com derivada numérica para diferentes valores da energia da impureza. 
Um formalismo de operadores de projeção para resolver o Hamiltoniano de Anderson

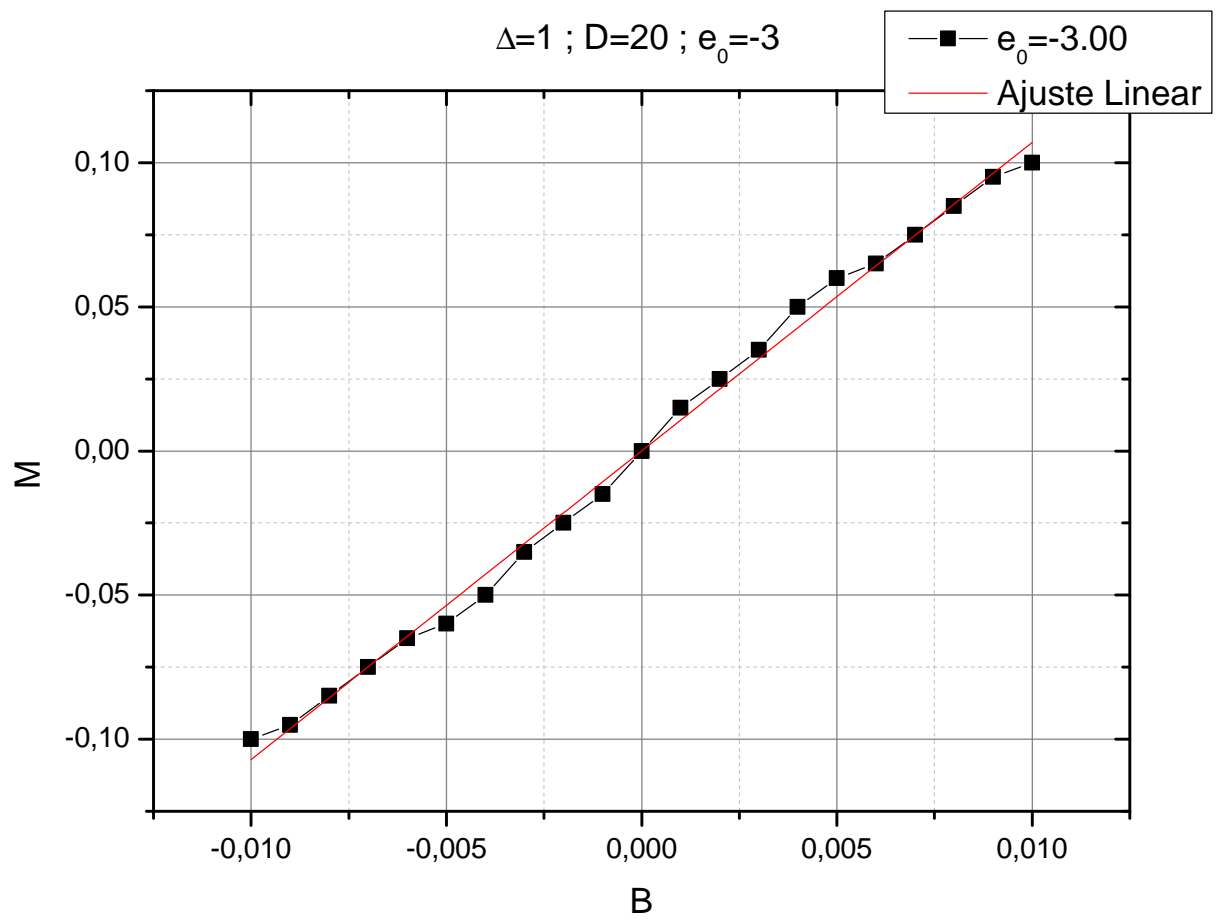

Figura 3.10: Magnetização da impureza como função do campo magnético externo, na região em que a magnetização possuiu um comportamento linear, para os mesmos parâmetros da figura $3.9 \operatorname{com} \epsilon_{0}=-3$. 


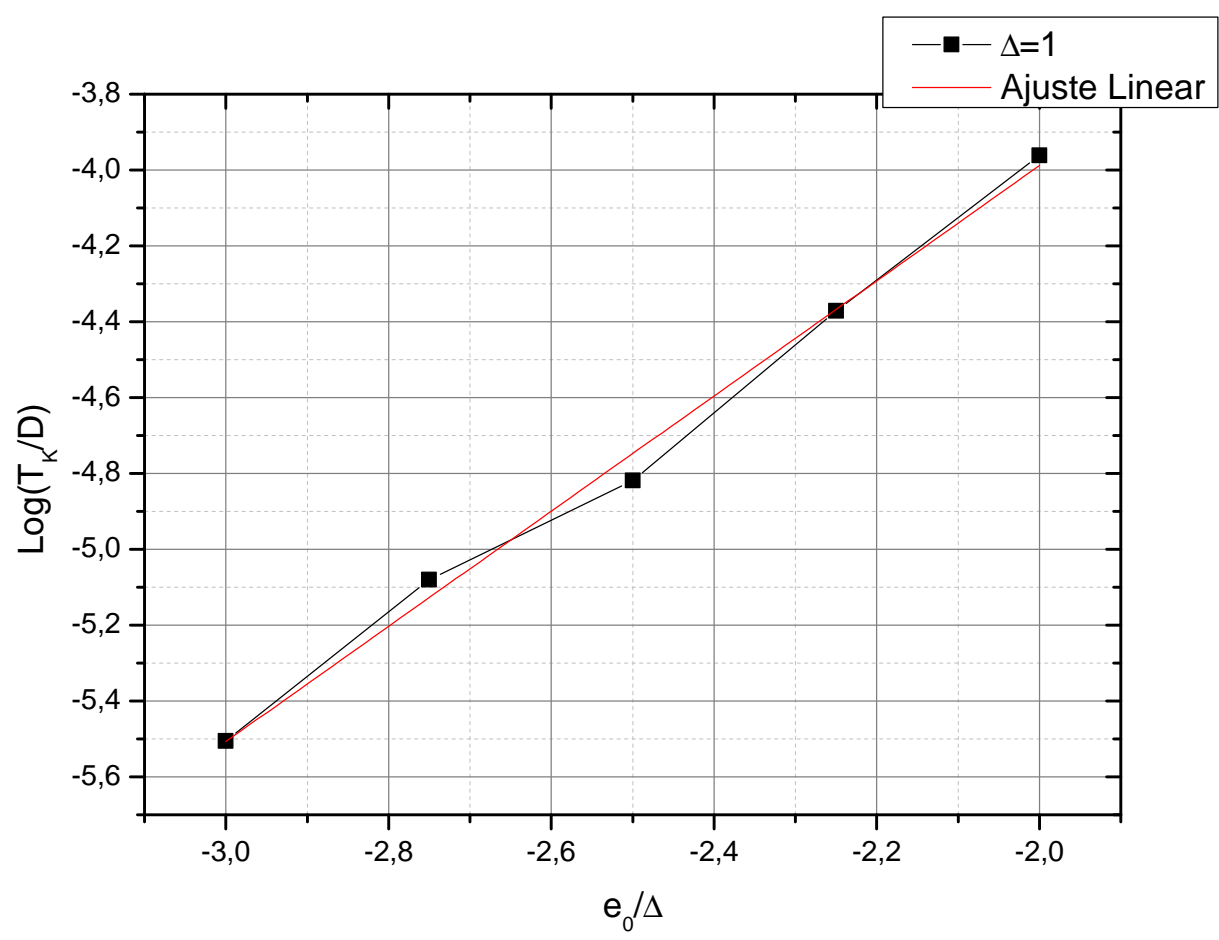

Figura 3.11: Evolução do logarítimo da razão da temperatura Kondo dividida pela semi-largura da banda, com a razão da energia da impureza dividida pela função de hibridização. 


\section{Conclusões e Perspectivas}

Nesta dissertação foi elaborado um formalismo que permite a determinação da energia do estado fundamental do Hamiltoniano da Impureza de Anderson utilizando operadores de projeção, os quais reduzem o espaço de Hilbert a uma única função e que requer a renormalização do Hamiltoniano, que, atuando neste subespaço, permite a obtenção da energia do estado fundamental de uma maneira autoconsistente.

Os resultados obtidos foram calculados considerando somente a contribuição dos termos diagonais, equação (2.4.8). Para valores dos parâmetros físicos típicos do problema Kondo, um cálculo da contribuição não diagonal seria da ordem de $10^{-4}$ menor que a contribuição diagonal para a correção da energia $\Delta E$ quando a impureza é conectada ao mar de Fermi.

Conhecendo a energia do estado fundamental é possível calcular a magnetização, a susceptibilidade magnética, a variação da população eletrônica em função da energia da impureza, a temperatura Kondo e a condutância (regra da soma de Friedel) (4) e (19).

É possível estender este formalismo para o estudo de sistemas mais complexos tais como estruturas de pontos quânticos, sistemas cuja densidade de estados depende fortemente da frequência na vizinhança do nível de Fermi ou sistemas com forte interação spin-órbita.

É conhecido o fato de que um sistema físico de dimensão infinita com interações Coulombianas locais, modelado pelo Hamiltoniano de Hubbard, pode ser mapeado num Hamiltoniano da impureza de Anderson autoconsistentemente. Embora os sistemas físicos reais não tem dimensão infinita, uma excelente aproximação para seu estudo é impor este mapeamento. Isto permite o estudo das propriedades de um sistema com interações Coulombianas no volume resolvendo o problema da impureza de Anderson equivalente. Este 
formalismo é chamado de Teoria de Campo Médio Dinâmico (DMFT), (8). Embora este tratamento requer das funções de Green correspondentes ao Hamiltoniano da Impureza de Anderson, as quais podem ser obtidas adotando uma filosofia equivalente a apresentada nesta dissertação, também é possível extender as ideias do DMFT no ambiente do cálculo da energia do sistema, assim como foi calculado nos capítulos anteriores. Esta seria sem dúvida uma extensão das ideias elaboradas nesta dissertação. 


\section{Referências Bibliográficas}

[1] O. MADELUNG, Introduction to Solid-State Theory, New York, Springer, (1981).

[2] N. W. ASHCROFT, N. D. MERMIN, Solid State Physics, Harcourt, (1976).

[3] J. P. FRANCK, F. D. MANCHESTER, DOUGLAS L. MARTIN, Proc. R. Soc. Lond. A, 263, (1961).

[4] A. C. HEWSON, The Kondo Problem to Heavy Fermions, Cambridge University Press, (1993).

[5] J. KONDO, Prog. Theor. Phys., 32, 37-49 (1964).

[6] D. GOLDHABER-GORDON, H. SHTRIKMAN, D. MAHALU, D. ABUSCH-MAGDER, U. MEIRAV, M. A. KASTNER, Nature, 391, 156158 (1998).

[7] J. R. SCHRIEFFER, P. A. WOLFF, Phys. Rev., 149, 491-492 (1966).

[8] A. Georges, G. KOtliar, W. KRAUth, M. J. ROZEnBERG, Rev. Mod. Phys., 68, 13-125 (1996).

[9] E. V. ANDA, G. CHIAPPE, C. A. BÜSSER, M. A. DAVIDOVICH, G. B. MARTINS, F. HEIDRICH-MEISNER, E. DAGOTTO, Phys. Rev. B, 78, 085308 (2008).

[10] K. G. WILSON, Rev. Mod. Phys., 47, 773-840 (1975).

[11] F. HEIDRICH-MEISNER, A. E. FEIGUin, E. DAGOTTO, Phys. Rev. B, 79, 235336 (2009).

[12] D. M. NEWNS, A. C. HEWSON, J. Phys. F: Metal Phys., 10, 2429-45 (1980).

[13] N. S. WINGREEN, Y. MEIR, Phys. Rev. B, 49, 11040-11052 (1994). 
Um formalismo de operadores de projeção para resolver o Hamiltoniano de Anderson

[14] N. E. BICKERS, Rev. Mod. Phys., 59, 845 (1987).

[15] L. TOSI, P. ROURA-BAS, A. M. LLOIS, L. O. MANUEL, Phys. Rev. B, 83, 073301 (2011).

[16] K. D. SCOTTE, U. SCHOTTE, Phys. Lett. A, 55, 38-40 (1975).

[17] D. N. ZUBAREV, Sov. Phys. Usp., 3, 320-345 (1960).

[18] H. BETHE, Z. Phys., 71, 205, (1931).

[19] D. C. LANGRETH, Phys. Rev., 150, 516-518 (1966).

[20] P. W. ANDERSON, J. Phys. C, 3, 2439 (1970). 